\title{
Review Article \\ Rheological Properties of Living Materials. From Cells to Tissues
}

\author{
C. VERDIER* \\ Laboratoire de Spectrométrie Physique, Université Joseph Fourier Grenoble I and CNRS (UMR5588), BP87-38402 Saint Martin d'Hères, France \\ (Received 10 February 2004; In final form 10 February 2004)
}

\begin{abstract}
In this paper, we review the role of the rheological properties at the cellular and macroscopic scale. At the cellular scale, the different components of the cell are described, and comparisons with other similar systems are made in order to state what kind of rheological properties and what constitutive equations can be expected. This is based on expertise collected over many years, dealing with components such as polymers, suspensions, colloids and gels. Various references are considered. Then we review the various methods available in the literature, which can allow one to go from the microscopic to the macroscopic properties of an ensemble of cells, in other words a tissue. One of the questions raised is: can we find different properties at the macroscopic level than the ones that we start with at the cellular level? Finally, we consider different biological materials which have been used and characterized, in order to classify them. Constitutive laws are also proposed and criticized. The most difficult part of modeling is taking into account the active part of cells, which are not just plain materials, but are living objects.
\end{abstract}

Keywords: Cell; Viscoelasticity; Biomechanics; Complex materials; Rheology; Viscoplasticity

\section{INTRODUCTION}

For many years, people have devoted their attention to the study of animal tissues (Fung, 1993a), and important issues have been raised. Finding constitutive relations for such media is not simple, because tissues can behave as elastic, plastic, viscoelastic or viscoplastic materials. One of the most important conclusion is that relating the microstructure (Larson, 1999) with its macroscopic nature is a fundamental problem which forms the basis of any continuum mechanics problem. The relevant sciences studying such aspects are rheology (Bird et al., 1987; Macosko, 1994; Larson, 1999), biomechanics (Fung, 1993a) or biorheology (journal with the same name). These three fields are actually very close to each other when it comes to dealing with biomaterials, and defining their minor differences here is not the purpose. One may say that generally we are interested in finding relationships between the applied forces and the relevant deformations or flows involved in problems dealing with living materials. Classical models (1D), which can be used and can depict the cytoplasm of a cell, are usually viscoelastic or viscoplastic ones. 3D-viscoelastic models can exhibit differential forms, or integral formulations (sometimes equivalent). Other models like viscoplastic ones can also be interesting because they allow us to deal with systems with cross-links, somehow close to gels; in particular, polymers and networks play a role inside the cytoplasm. So, at a certain level, we may consider that the size of the system studied $\left(L_{\mathrm{s}}\right)$ is large enough $L_{\mathrm{s}} \gg L_{\mathrm{e}}$ (where $L_{\mathrm{e}}$ is the size of an element at the microscopic level) so that the system can behave in a macroscopic way and can obey a constitutive equation. We are precisely discussing here the possibility to go from a microrheological to a macrorheological measurement. This will be an important part of the second chapter, where we will review the different methods available to investigate the local microrheology of a biological system. Indeed, recent advances in this field now allow a wide range of data to be obtained using sophisticated techniques coming from physics. Of course, before going deeper into this sort of analysis, a careful definition of the different elements present inside the cell will be needed. Comparisons with the different classical systems studied in rheology (polymers, suspensions, gels, etc.) will be made. We will also see that problems involving interfaces between domains are also relevant here due to

*Tel.: + 33-4-76-63-59-80. Fax: +33-4-76-63-54-95. E-mail: verdier@ujf-grenoble.fr 
the presence of membranes, which play a particular (if not major) role in the interactions between cells through the presence of proteins. Such topics about membranes are well discussed (Lipowsky and Sackmann, 1995) and can account for the diffusivity of proteins along membranes as well as their stability.

In the third part, methods for going from the cell to the tissue will be reviewed. The common methods from mechanics, like homogenization (Sanchez-Palencia, 1980), discrete homogenization (Caillerie et al., 2003), tensegrity (Ingber et al., 1981), effective medium theories (Choy, 1999), and ensemble average theories (Batchelor, 1970) will be discussed. All of these methods have been used in the frame of standard materials (composites, porous media, etc.) but, unfortunately, are seldom used in the field of biological systems, although they would provide a better understanding of the systems. The tensegrity method seems to be quite suitable to cells, and has been the method studied most extensively. It has the advantage of exhibiting simple equivalents to the cell microstructure in terms of sticks and elastic strings, looking very much like a network of actin filaments and microtubules, with intermediate filaments linked to each other.

Finally, tissues will be considered; in particular, the viscoelastic (viscoplastic) relations, which have been proposed in the literature, will be reviewed. The difficulty in the determination of constitutive equations is that, to be able to have access to the 3D law, one needs to perform various simple tests, in particular, in shear and elongation, which are not so simple on actual biological tissues. In some cases, human tissues can be used, but sometimes it is not possible to carry out experiments, and so there are few data available. Nevertheless, an attempt will be made to classify examples, which have been known for some time (Fung, 1993a), but recent data will also be presented.

The aim of the present work is not to cover this field in an exhaustive manner, but rather to give access to data, references, constitutive equations of living materials and the interrelationship between microrheology and macrorheology for those who use biological systems.

\section{RHEOLOGICAL MODELS}

Rheology is defined as the science of flowing materials, or what are the stresses that one needs to apply to achieve a certain rate of deformation in a given material. This concept relies on continuum mechanics theories, where references are quite numerous (see, for example, Sedov, 1975; Fung, 1993b). After introducing these concepts, one has to treat the case of the linear elastic solid and the viscous fluid. The combination of these models leads to the concept of viscoelasticity, and this is where we will start, considering the Maxwell fluid in one dimension. Then we will show

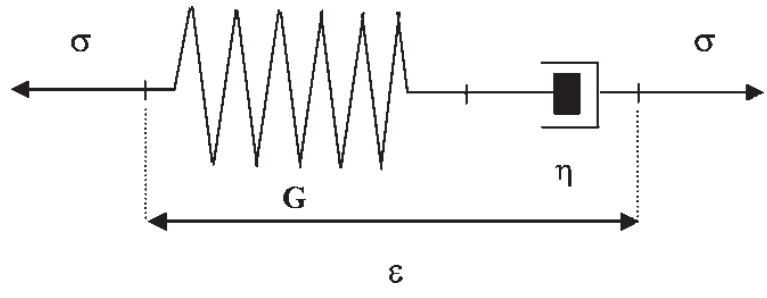

FIGURE 1 The Maxwell element.

how to construct more sophisticated models. The concept of viscoplasticity, which is also seen to be very important (Schmidt et al., 2000a), will also be described.

\section{Simple One-dimensional Model}

As a starting point, we introduce the concept of the onedimensional viscoelastic Maxwell element. Although it is a simple example, it can be very useful to understand a lot of the concepts, which will be presented next, and it will also be used to introduce definitions. It consists of a spring and a dashpot in series (Fig. 1).

The constitutive equation associated with such a model is derived when considering strains encountered by the different elements, the spring (rigidity $G$, strain $\varepsilon_{1}$, stress $\sigma=G \varepsilon_{1}$ ) and the dashpot (viscosity $\eta$, strain $\varepsilon_{2}$, stress $\sigma=\eta \dot{\varepsilon}_{2}$ ). The sum of the strains in the two elements $\varepsilon=\varepsilon_{1}+\varepsilon_{2}$ is related to the total stress $\sigma$. By differentiation of the previous equation, we find the following constitutive equation:

$$
\sigma+\lambda \dot{\sigma}=\eta \dot{\varepsilon}
$$

where $\lambda=\eta / G$ is the relaxation time. This expression is the differential form of the model and defines already a first class of models of this kind. On the contrary, integral models, when they exist, can be quite useful. In the case of the Maxwell model, by simple integration of Eq. (1), one can derive the integral formulation giving the stress explicitly in terms of the strain $\varepsilon(t)$ :

$$
\sigma(t)=\int_{-\infty}^{t} G \exp \left(-\left(t-t^{\prime}\right)\right) \dot{\varepsilon}\left(t^{\prime}\right) \mathrm{d} t^{\prime}
$$

The advantage of this form is that stresses are related to the strain history. Indeed elasticity requires the material to recover its initial shape or at least some shape close to it. Therefore, the kernel function $G(t)=G \exp (-t / \lambda)$ is the relaxation function and measures how much memory is retained by the material. ${ }^{\dagger}$ For recent past times, it remembers a lot, whereas for old times, it does not recall much. This function is also the solution of Eq. (1) with no right-hand side. When $\dot{\varepsilon}$ is a constant, the right-hand side is also a constant and the solution is simply $\sigma(t)=\eta \dot{\varepsilon}(1-\exp (-t / \lambda))$.

\footnotetext{
${ }^{\dagger}$ Note: we could also discuss the model where a spring and dashpot are in parallel (Kelvin-Voigt model, viscoelastic solid). In such a case, the 1D equation simply becomes $\sigma=G \varepsilon+\eta \dot{\varepsilon}$, which gives an explicit formula for the stress $\sigma$. Conversely, the deformation $\varepsilon(t)$ can then be calculated in terms of $\sigma(t)$ similarly to Eq. (2). The kernel is called the compliance and is named $J(t)$, to be compared with the relaxation function $G(t)$.
} 
The steady state solution $\sigma_{p}=\eta \dot{\varepsilon}$ defines the viscosity as $\eta=\sigma_{p} / \dot{\varepsilon}$ which is a constant in this case.

The function $G(s)$ has been presented in this case where only one relaxation time is given, but there might be cases where more than one relaxation time is needed, as in the case of the dynamics of polymeric materials (Larson, 1988; Macosko, 1994). The simplest way to generalize this formulation is through the introduction of a sum of exponentials:

$$
G(t)=\sum_{i=1}^{n} G_{i} \exp \left(-t / \lambda_{i}\right)
$$

We will see now how these assumptions can help to obtain accurate data, in particular when spectra are obtained over decades in time or frequency. In particular, it can be observed that time or frequency information is equivalent or complementary. So far we only paid interest to timedependent behavior through relation (2). So let us now look at frequency-dependent data.

Another typical experiment which one can easily perform on common rheometers (Walters, 1975) is dynamic testing. This principle is very important when considering small deformation theory. Indeed, when a material is sheared (but it might also be elongated) by imposing a sinusoidal deformation $\gamma=\gamma_{0} \sin (\omega t)$, such that $\gamma_{0} \ll 1$, we may assume that the resulting stress $\tau$ is also a sinusoidal function $\tau=\tau_{0} \sin (\omega t+\delta)=$ $\tau_{0} \cos (\delta) \sin (\omega t)+\tau_{0} \sin (\delta) \cos (\omega t)$. This gives rise to a modulus $G^{\prime}=\tau_{0} \cos (\delta)$, in phase with the deformation, and another part $G^{\prime \prime}=\tau_{0} \sin (\delta)$ in phase with the rate of deformation. These two moduli are therefore called the elastic modulus $\left(G^{\prime}\right)$ and the loss or viscous modulus $\left(G^{\prime \prime}\right)$ because the latter is related to viscous dissipation in the sample tested. Quite often this information is very useful because it enables one to see how much elasticity exists compared to viscous losses. The angle $\delta$ contains this information through the ratio of the two moduli: $\tan (\delta)=$ $G^{\prime \prime} / G^{\prime}$. These expressions are often described in the complex domain, and $G^{*}=G^{\prime}+i G^{\prime \prime}$ is defined as the complex modulus whereas $\eta^{*}=\eta^{\prime}-i \eta^{\prime \prime}=G^{\prime \prime} / \omega-$ $i G^{\prime} / \omega$ is the complex viscosity. The complex viscosity is related to the previous relaxation modulus $G(t)$ and to $G^{*}(\omega)$ through:

$$
\eta^{*}(\omega)=G^{*}(\omega) / i \omega=\int_{0}^{\infty} G(t) \exp (-i \omega t) \mathrm{d} t .
$$

Let us go back to the Maxwell model, and see the predictions using $G(t)=G \exp (-t / \lambda)$. This gives the following relation for the complex modulus $G *(\omega)$ and dynamic $G^{\prime}, G^{\prime \prime}$ :

$$
\begin{aligned}
G^{*}(\omega) & =G \frac{i \lambda \omega}{1+i \lambda \omega}, \quad G^{\prime}(\omega)=G \frac{\lambda^{2} \omega^{2}}{1+\lambda^{2} \omega^{2}}, \\
G^{\prime \prime}(\omega) & =G \frac{\lambda \omega}{1+\lambda^{2} \omega^{2}} .
\end{aligned}
$$

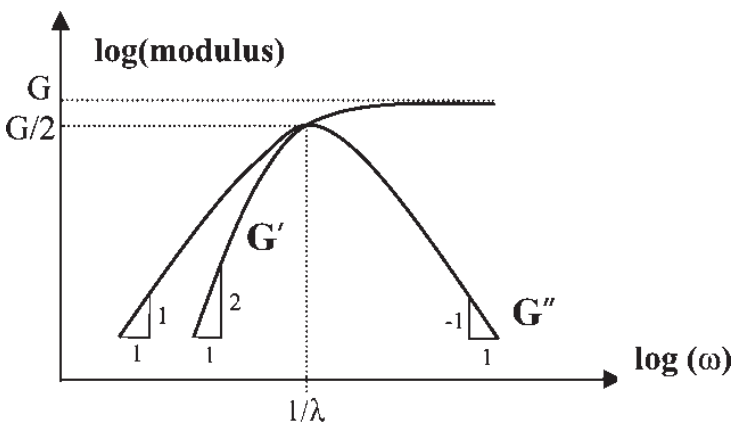

FIGURE 2 Dynamic moduli $\left(G^{\prime}, G^{\prime \prime}\right)$ in the case of the Maxwell model.

These behaviors are easily seen in Fig. 2 for the real moduli $G^{\prime}$ and $G^{\prime \prime}$ :

In reality, most systems, such as polymeric ones or suspensions do not behave like this, as previously described, and exhibit multiple relaxation effects. A discrete sum of relaxation modes can then be proposed, as in Eq. (3), for which the corresponding formulation in terms of $G^{*}(\omega)$ is, for $n$ relaxation modes:

$$
G^{*}(\omega)=\sum_{i=1}^{n} G_{i} \frac{i \lambda_{i} \omega}{1+i \lambda_{i} \omega} .
$$

In general, the typical curves found for polymeric materials are as shown in Fig. 3. There are four regimes to be analyzed as follows. The low-frequency regime corresponds to typical slopes of 2 and 1 for $G^{\prime}$ and $G^{\prime \prime}$, respectively, which is the Newtonian (fluid-like) behavior. As the frequency increases, the rubbery plateau is observed corresponding to a plateau modulus $\left(G_{N}^{0}\right)$ for $G^{\prime}$. Again we increase the frequency, and the two curves are close to each other with similar slopes (typically 0.6), which is a characteristic of the glass transition, until we arrive at the solid-like state at very high frequencies. In Fig. 3, we also show the behavior of a viscoplastic fluid, but this feature could also be observed for viscoelastic solids (cross-linked polymers for example). These materials cannot flow even at very low shears, either because strong links exist within the microstructure or simply because weak links exist (i.e. physical gels) which would mean breaking the system to make them flow.

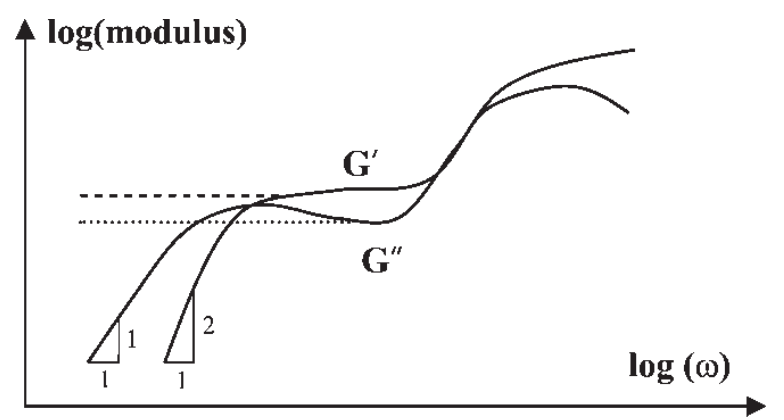

FIGURE 3 Typical curves $\left(G^{\prime}, G^{\prime \prime}\right)$ for a complex fluid and a viscoplastic material (different low frequency regime: dotted lines, solidlike behavior). 
These ideas related to the microstructure will be discussed later.

As a final sophistication of the model, it is easy to see that the relaxation function proposed in Eq. (3) containing elements $\left(G_{i}, \lambda_{i}\right)$ can be extended (Baumgaertel and Winter, 1989) to a continuous function or so-called continuous relaxation spectrum $H(\lambda)$ through the following formula:

$$
G(t)=\int_{0}^{\infty} \frac{H(\lambda)}{\lambda} \exp (-t / \lambda) \mathrm{d} \lambda .
$$

Examples of the use of such models have been treated in particular in the case of molten polymers (Baumgaertel and Winter, 1989; Jackson and Winter, 1996; Verdier et al., 1998). With these models, it is possible to investigate, as an inverse problem, the chain length distribution of polymeric systems, and provide a valuable tool for understanding the microstructure of the system.

\section{Three-dimensional Models}

\section{Going from 1D to 3D Models}

When formulating three-dimensional constitutive equations, special attention needs to be paid to the principle of frame indifference or objectivity principle: operators or tensors need to satisfy rules so that constitutive equations remain valid in any reference frame. In particular, generalization of Eq. (1) could be thought of by just replacing the quantities $\sigma$ and $\dot{\varepsilon}$ by their counterparts, i.e. the tensors $\boldsymbol{\sigma}$ and $\mathbf{D}$, where $\mathbf{D}$ is the symmetric part of the velocity gradient tensor $\operatorname{grad} \mathbf{v}=\nabla \mathbf{v}$, and $\mathbf{v}$ is the velocity vector. This is not possible because we are interested in frame-indifferent constitutive equations. So the derivative of $\boldsymbol{\sigma}$ needs more attention. In fact this is all we need to look at to generalize Eq. (1) because in fact $\boldsymbol{\sigma}$ and $\mathbf{D}$ are already objective tensors. One of the possibilities for an objective time derivative of $\boldsymbol{\sigma}$ is the so-called upper-convected derivative, denoted by $\underset{\nabla}{\boldsymbol{\sigma}}$ :

$$
\stackrel{\nabla}{\boldsymbol{\sigma}}=\frac{\partial \boldsymbol{\sigma}}{\partial t}+\mathbf{v} \cdot \nabla \boldsymbol{\sigma}-(\nabla \mathbf{v})^{T} \cdot \boldsymbol{\sigma}-\boldsymbol{\sigma} \cdot \nabla \mathbf{v}
$$

where $\nabla \mathbf{v}$ has components $\partial v_{i} / \partial x_{j}$ in a usual Cartesian coordinate system. Other derivatives such as the lowerconvected derivative and the corotational derivatives, or combinations of these also exist but will not be discussed here. Nevertheless they can be found in appropriate textbooks (Bird et al., 1987; Larson, 1988; Macosko, 1994). Now we let $\boldsymbol{\sigma}=-p \mathbf{I}+\boldsymbol{\sigma}^{\prime}$, which enables the definition of an isotropic pressure term $(p)$. Usually, terms involving isotropic components will be included in this part, but we will concentrate only on the extra-stress term $\boldsymbol{\sigma}^{\prime}$, and we will now drop the primes for simplicity. In shear motions, the interesting components will include only shear terms and so $p$ has no effect. In elongation, the attention will be focused on stress differences, thus eliminating the pressure term.

The final 3D constitutive equation for viscoelastic medium now reads:

$$
\boldsymbol{\sigma}+\lambda \stackrel{\nabla}{\boldsymbol{\sigma}}=2 \eta \mathbf{D}
$$

It is a frame-indifferent constitutive equation, which globally retains the physical basis of the viscoelastic fluid, i.e. at small times $t \ll \lambda$, the material behaves elastically, and at the longer times $t \gg \lambda$, it behaves as a liquid and is able to flow.

An equivalent integral formulation of Eq. (9) exists (to within the addition of a pressure $p$ ), and is given by:

$$
\boldsymbol{\sigma}=\int_{-\infty}^{t} G / \lambda \exp \left(-\left(t-t^{\prime}\right) / \lambda\right) \mathbf{B}\left(t, t^{\prime}\right) \mathrm{d} t^{\prime}
$$

where the modulus $G$ and relaxation time $\lambda$ have been defined previously, while the Finger tensor $\mathbf{B}\left(t, t^{\prime}\right)$ is introduced as a strain measurement from a previous configuration $\mathbf{x}^{\prime}$ (at time $t^{\prime}$ ) to a new position $\mathbf{x}=\mathbf{x}\left(\mathbf{x}^{\prime}, t, t^{\prime}\right)$ at time $t$. The relative deformation gradient is $\mathbf{F}\left(t, t^{\prime}\right)=$ $\partial \mathbf{x} / \partial \mathbf{x}^{\prime}$ and $\mathbf{B}=\mathbf{F} \mathbf{F}^{T}$.

\section{The General Elastic Solid}

Formulation (10) is actually just a generalization of the elasticity of a material (such as rubber) when large deformations are involved. In particular, if we go back to elasticity theory for a moment, we have precisely:

$$
\sigma=-p \mathbf{I}+G \mathbf{B}
$$

where $G$ is the shear modulus, and $p$ a general term which is needed for generality.

This relationship works well for rubbers and is generalized by adding extra powers of $\mathbf{B}$, including the invariants: $\boldsymbol{\sigma}=a_{0} \mathbf{I}+a_{1} \mathbf{B}+a_{2} \mathbf{B}^{2}+\ldots$ These power terms are reduced by making use of the Cayley-Hamilton theorem, which leads to:

$$
\boldsymbol{\sigma}=b_{0} \mathbf{I}+b_{1} \mathbf{B}+b_{2} \mathbf{B}^{-1}
$$

where the $b_{i}$ 's are functions of the first and second invariants of $\mathbf{B}, \mathrm{I}_{\mathrm{B}}=\operatorname{tr}(\mathbf{B})$ and $\mathrm{II}_{\mathrm{B}}=1 / 2\left\{(\operatorname{tr} \mathbf{B})^{2}-\right.$ $\left.\operatorname{tr}\left(\mathbf{B}^{2}\right)\right\}$. This formulation is also known as the Mooney-Rivlin form and is interesting for going beyond the first viscoelastic relations such as Eq. (10). It can also be generalized again in the context of strain-energy functions. Again, another formulation for the investigation of general elastic solids is:

$$
\boldsymbol{\sigma}=-p \mathbf{I}+2 \partial W / \partial \mathrm{I}_{\mathrm{B}} \mathbf{B}-2 \partial W / \partial \mathrm{II}_{\mathrm{B}} \mathbf{B}^{-1}
$$

where $W\left(\mathrm{I}_{\mathrm{B}}, \mathrm{II}_{\mathrm{B}}\right)$ is the strain-energy function and has been used extensively (Humphrey, 2003) for the study of biological materials. 


\section{The Generalized Newtonian Fluid}

One can recall the relationship for an incompressible Newtonian fluid, which is simply:

$$
\boldsymbol{\sigma}=-p \mathbf{I}+2 \eta \mathbf{D} .
$$

Again, it has been shown that this relationship can give rise to a more general form, which is still frame-invariant, because of the objectivity of the tensor $\mathbf{D}$ :

$$
\boldsymbol{\sigma}=-p \mathbf{I}+\eta_{1} 2 \mathbf{D}+\eta_{2}(2 \mathbf{D})^{2} .
$$

This has been called the Reiner-Rivlin fluid. The coefficient $\eta_{1}$ is a viscosity and $\eta_{2}$ is another coefficient, both depending on the second $\left(\mathrm{II}_{\mathrm{D}}\right)$ and third $\left(\mathrm{III}_{\mathrm{D}}\right)$ invariants of $\mathbf{D}$. This generalized fluid is very important because it is the first law to be able to predict a non-zero stress difference $\sigma_{22}-\sigma_{33}$, although the first stress difference $\sigma_{11}-\sigma_{22}$ is zero in simple shear flows. Finally, people usually assume that $\eta_{2}=0$, and the dependence of $\eta_{1}$ as a function of $\mathrm{II}_{\mathrm{D}}$ can be chosen so that a good description of most polymeric systems and suspensions is obtained in shear, as will be discussed later. Typical behaviors observed are the shear-thinning fluids (see Fig. 4 below), or in some cases shear thickening effects such as those observed in suspensions. Shear-thinning fluids are well described by power-law models,

$$
\eta_{1}=m\left|\mathrm{II}_{\mathrm{D}}\right|^{(n-1) / 2} \quad \text { and } \quad \eta_{2}=0
$$

or by the Yasuda-Carreau model:

$$
\frac{\eta_{1}-\eta_{\infty}}{\eta_{0}-\eta_{\infty}}=\frac{1}{\left[\left(1+\left(\lambda \sqrt{\left|\mathrm{II}_{2 \mathrm{D}}\right|}\right)^{a}\right)\right]^{(1-n) / a}} \text { and } \eta_{2}=0 .
$$

This model has a zero-shear viscosity $\eta_{0}$, a limiting viscosity $\eta_{\infty}$ at high shear rates, a relaxation time $\lambda$, a power-law behavior in the intermediate regime, and another adjustable parameter $a$. It is well adapted for polymers and polymer solutions.

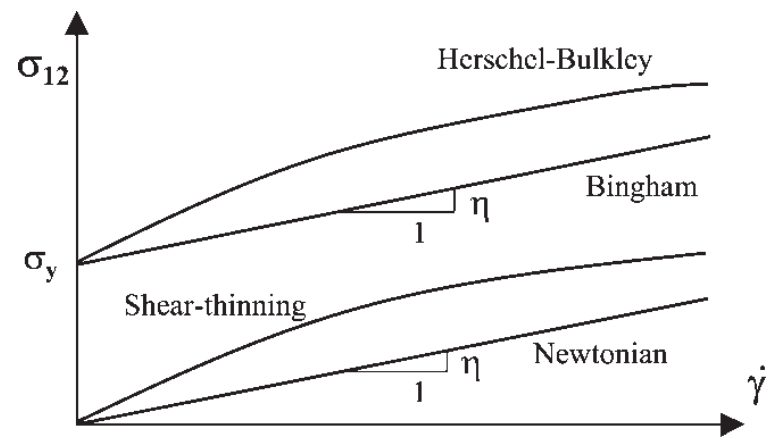

FIGURE 4 Typical curves $\sigma_{12}(\dot{\gamma})$ for shear thinning fluids and viscoplastic ones.

\section{The Viscoplastic Fluid}

After defining the Reiner-Rivlin fluid, it is simple to introduce a relationship for the viscoplastic fluid. This material can flow only when stresses are higher than a certain threshold, called the Yield stress $\left(\sigma_{y}\right)$. Below this value, the material will behave in an elastic manner. The proposed constitutive equation (known as Bingham model) is as follows:

$$
\begin{aligned}
& \text { - }\left|\mathrm{II}_{\sigma}\right|<\sigma_{y}^{2} \quad \boldsymbol{\sigma}=G \mathbf{B} \quad \text { or } \quad \mathbf{D}=0 \\
& \text { - }\left|\mathrm{II}_{\sigma}\right|>\sigma_{y}^{2} \quad \boldsymbol{\sigma}=\left(\eta+\frac{\sigma_{y}}{\sqrt{\left|\mathrm{II}_{2 \mathrm{D}}\right|}}\right) 2 \mathbf{D} .
\end{aligned}
$$

In this relation, $\mathrm{II}_{\sigma}$ is the second invariant of the stress tensor, where the isotropic pressure term is omitted. $\mathrm{II}_{2 \mathrm{D}}$ is also the second invariant of the tensor 2D. In a classical simple shearing test, at constant strain rate $\dot{\gamma}$, these relations would simply give $\sigma_{12}<\sigma_{y}, \sigma_{12}=G \gamma$ (or $\dot{\gamma}=0$ ); and for $\sigma_{12}<\sigma_{y}, \sigma_{12}=\sigma_{y}+\eta \dot{\gamma}$.

Finally, let us note that a few other models of this kind exist (Macosko, 1994), for example, the widely used Herschel-Bulkley model:

$$
\begin{aligned}
& \text { - }\left|\mathrm{II}_{\sigma}\right|<\sigma_{y}^{2} \quad \boldsymbol{\sigma}=G \mathbf{B} \text { or } \mathbf{D}=0 \\
& \text { - }\left|\mathrm{II}_{\sigma}\right|>\sigma_{y}^{2} \\
& \boldsymbol{\sigma}=\left(m\left|\mathrm{II}_{2 \mathrm{D}}\right|^{(n-1) / 2}+\frac{\sigma_{y}}{\sqrt{\left|\mathrm{II}_{2 \mathrm{D}}\right|}}\right) 2 \mathbf{D} .
\end{aligned}
$$

In this formula, $m$ is a constant with the proper unit, and $n$ is a dimensionless parameter related to the slope of the shear stress vs. shear rate curve (Fig. 4).

\section{More Complex Viscoelastic Laws}

Let us now go back to more general forms of Eqs. (9) and (10) representing viscoelastic materials. The integral form of Eq. (10) can be extended to any memory function $G(t)$, as given for instance by Eq. (3) as a sum of exponentials. The only conditions are that this function $G(t)$ should be finite for $t=0$, decreasing $G^{\prime}(t)<0$ and convex $G^{\prime \prime}(t)>$ 0 . The second extension is the use of a strain-energy function. These two extensions give rise to the so-called K-BKZ model (Larson, 1988), in its factorized version:

$$
\begin{aligned}
\boldsymbol{\sigma}= & \int_{-\infty}^{t} 2 G\left(t-t^{\prime}\right) \\
& \times\left[\frac{\partial \mathrm{U}}{\partial \mathrm{I}_{\mathrm{B}}} \mathbf{B}\left(t, t^{\prime}\right)-\frac{\partial \mathrm{U}}{\partial \mathrm{II}_{\mathrm{B}}} \mathbf{B}^{-1}\left(t, t^{\prime}\right)\right] \mathrm{d} t^{\prime}
\end{aligned}
$$

where $u\left(\mathrm{I}_{\mathrm{B}}, \mathrm{II}_{\mathrm{B}}\right)=G\left(t-t^{\prime}\right) \quad \mathrm{U}\left(\mathrm{I}_{\mathrm{B}}, \mathrm{II}_{\mathrm{B}}\right)$ is the kernel energy function. One may also use more general functions instead of the derivatives of $U$ in front of the tensors $\mathbf{B}$ and $\mathbf{B}^{-1}$ (see, for example, Bird et al., 1987). These relations have been shown to be quite efficient for describing 
the nonlinear properties of complex systems in particular in elongation experiments (Wagner, 1990).

Finally, the extension of the constitutive equations of the differential type (9) is also possible and provides a good description of some complex fluids. The generalized forms of Eq. (9) can be written as:

$$
\boldsymbol{\sigma}+\mathbf{f}(\boldsymbol{\sigma}, \mathbf{D})+\lambda \stackrel{\nabla}{\boldsymbol{\sigma}}+\mathbf{g}(\boldsymbol{\sigma})=2 \eta \mathbf{D}
$$

where $\mathbf{f}(\boldsymbol{\sigma}, \mathbf{D})$ and $\mathbf{g}(\boldsymbol{\sigma})$ are nonlinear functions, provided in Macosko (1994), which correspond to various models, in particular, the Johnson and Segalman (1977), White and Metzner (1963), Giesekus (1966), Leonov (1976) and Phan-Thien and Tanner (1977) models.

To conclude, we summarize the results by noticing that the complexity of all these models is clear, but all the parameters used can be found using separate experiments (shear and elongation) and they finally provide a good comparison with experimental data including complex flows. The next part will now give examples of complex materials and their related microstructure, and the laws that describe them.

\section{Anisotropic Materials}

Most of the relations above have assumed that the systems are isotropic, i.e. that the relationships do not depend on the orientation of the sample tested. Nevertheless, many materials (composites, suspensions of rod-like particles, liquid crystals, tissues, etc.) can be anisotropic, even at rest. We will not enter too much into this discussion because there are adequate references in the literature (Boehler, 1983; Smith, 1994). For example, in elasticity theory, the stress-strain relationship which leads to Eq. (11) in small deformations $\boldsymbol{\sigma}=G \boldsymbol{\varepsilon}$ (neglecting $p$, and where $\boldsymbol{\varepsilon}$ is the small deformation strain tensor) can be generalized to the anisotropic case by letting $G$ be a fourth-order tensor. In such a case there is not only one elastic (Young) modulus $E$, but most likely there should be one in every direction, and similarly for the Poisson's coefficient $\nu$. For fluids, similar relationships can also be provided, when the fluid has preferred directions (e.g. liquid crystals), thus ruling out the previously mentioned relations.

\section{Some Typical Rheological Properties of Complex Materials}

There are a few complex systems, which are relevant to the study of animal or human cell, which need to be investigated further since we are interested in a complex system made of polymers, suspensions, gels, micellar systems. The microstructure of these systems is very important for the elaboration of constitutive equations, such as those described previously. Let us first review the rheological properties of a few of these systems, as summarized in Table I.

\section{Polymers and Polymer Solutions}

A few properties have already been proposed. Polymers are viscoelastic or may become viscoplastic in some cases (polymer gels). They are present inside the cell and are named proteins. They play a fundamental role for many cell functions and are crucial in cell-cell interactions. Their main features are as follows:

- Zero shear viscosity is a function of the molecular weight (length of the chains).

- Time-temperature superposition principle: curves at different temperatures can be shifted and superposed onto similar ones to cover larger decades in frequencies.

- Shear thinning behaviors, Eqs. (16) and (17), with exponents $n=0.3-0.8$ typically.

- $\left(G^{\prime}, G^{\prime \prime}\right)$ spectra can be best fitted using Eqs. (6) and (7) like that in Fig. 3.

- Non-zero first normal stress difference $\left(N_{1}=\sigma_{11}-\right.$ $\left.\sigma_{22}>0\right)$ and negative second normal stress difference $\left(N_{2}=\sigma_{22}-\sigma_{33}<0\right)$.

- Elongational properties are often predicted using integral laws such as Eq. (20). A typical elongational curve is shown in Fig. 5 below, where $\eta_{E}^{+}(t)=$ $\left(\sigma_{11}-\sigma_{22}\right) / \dot{\varepsilon}$.

There are also other physical models which have been used in the past, like the theory of reptation (de Gennes, 1979) or the tube model (Doi and Edwards, 1986), arising from considerations based on local friction coefficients. These theories have the advantage that they arise from microscopic considerations. Their predictions are useful, in particular, in dynamic testing.

The basic microstructure of a polymer network consists of chains intermingled with each other (entanglements) with a few weak reticulation points, as well as loops or dangling ends (de Gennes, 1979). If one wants to relate the microstructure of the polymer chains under flow or deformation, it is quite difficult to do since it involves very small scales (nanometers). Therefore few techniques exist, but recently fluorescence images using markers have been shown to be useful tools for investigating the dynamics of polymeric chains, for example, when stretching DNA molecules (Perkins et al., 1995).

Elastomers are slightly different and may be considered as viscoelastic solids, in particular, because they cannot flow at very low rates (Fig. 3). Therefore, they can be considered to be viscoplastic fluids and obey Eqs. (18) and (19). This is mainly due to strong links (covalent sometimes) associating polymer chains thus creating a network which behaves elastically over a wide range of rates. Their microstructure looks something like a regular net, at very small scales again (nanometers). As the frequency is increased, they undergo a glassy transition where moduli $G^{\prime}$ and $G^{\prime \prime}$ behave as $\omega^{n}$, where $n$ is an exponent whose value is close to 0.6. This behavior has 
RHEOLOGICAL PROPERTIES OF LIVING MATERIALS

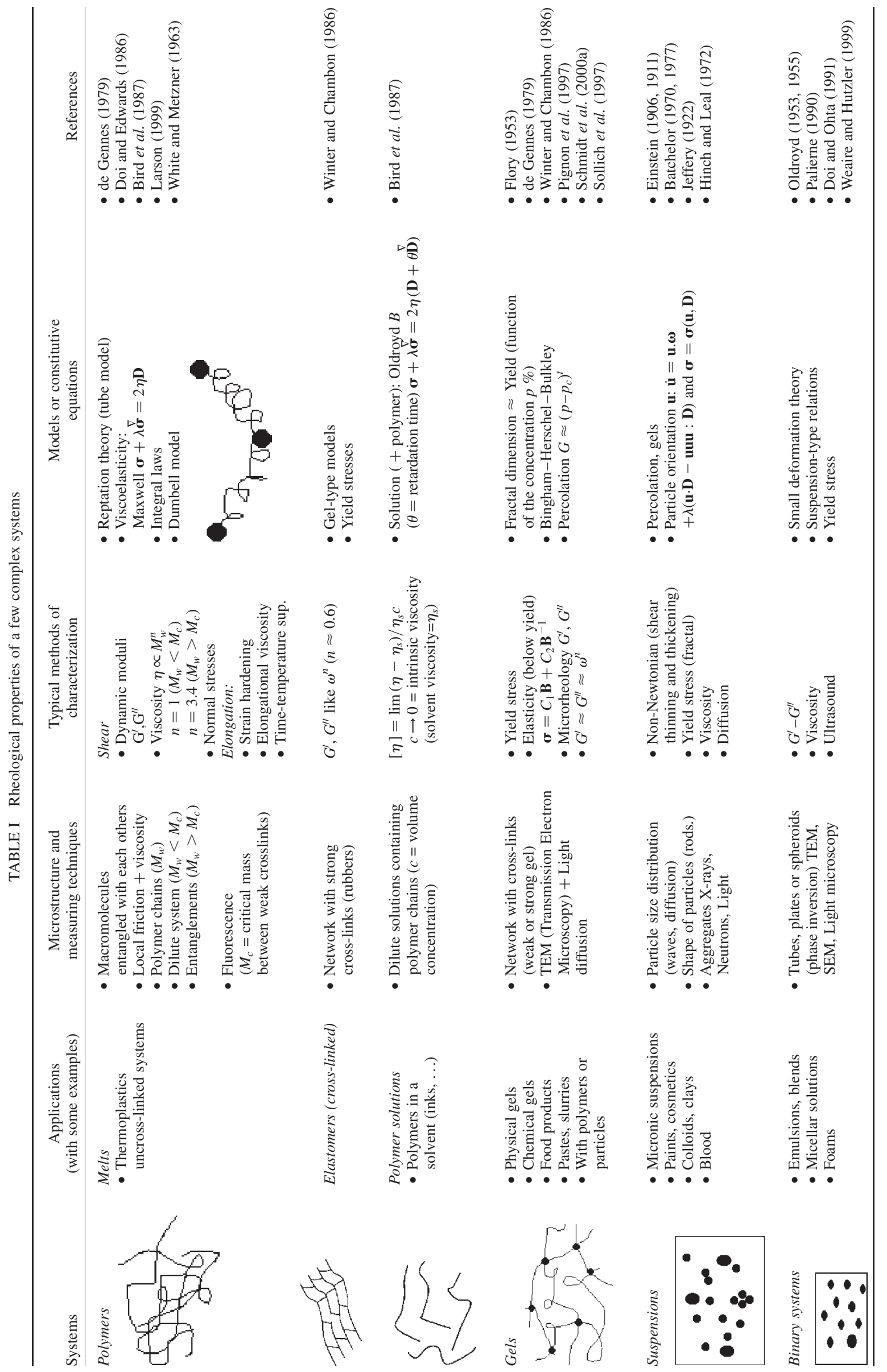




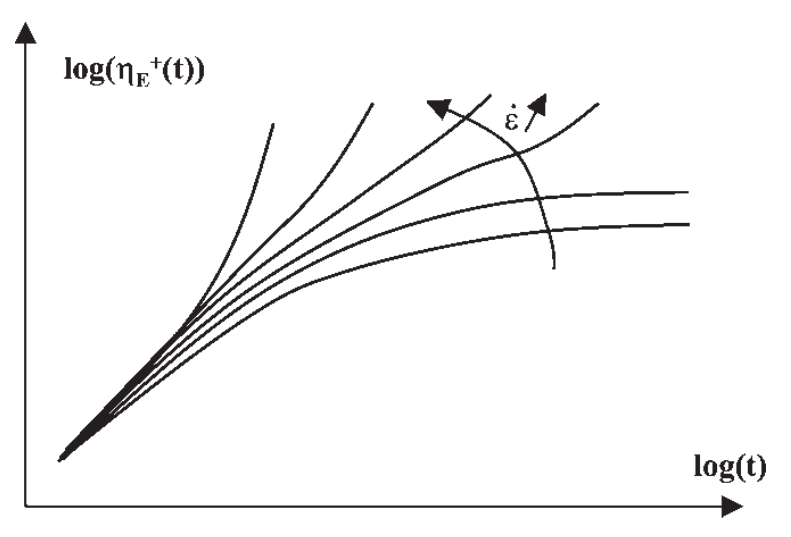

FIGURE 5 Elongational viscosity as a function of time $(\dot{\varepsilon}=$ constant $)$. The two curves at the lowest $\dot{\varepsilon}$ show a plateau, therefore the steady state exits. In the other cases $(\dot{\varepsilon}>1 / 2 \lambda)$, there is strain hardening, i.e. the viscosity increases exponentially.

been observed in gel-like systems also (Winter and Chambon, 1986) for cross-linking polymers close to the gel point.

Polymer solutions are solutions containing polymers in a solvent and do not exhibit entanglements in this regime. They may be considered to have two components, one being the solvent (which is viscous with constant viscosity $\eta_{s}$ ) and the other one being the polymer with viscoelastic properties like in 3D Maxwell's equation (9). The resulting equation (Table I) is called the Olroyd-B fluid, which has another characteristic time, called the retardation time $\theta$. Sometimes, the intrinsic viscosity $[\eta]=\left(\eta-\eta_{s}\right) / \eta_{s} c \quad($ as $c \rightarrow 0)$ is used to separate the effect of the viscosity of the polymer (volume concentration $c$ ) as compared to that of the solvent. The addition of a few percent of polymer to a solvent is particularly interesting for example for changing the breakup properties of jets, for reducing drag, for increasing tackiness (Verdier and Piau, 2003), etc.

\section{Suspensions}

The field of suspensions is quite large, because it can describe particulate suspensions, but can also lead to fluid-fluid suspensions called emulsions, and all kinds of systems including deformable objects in a fluid. For example, blood is a mixture of white and red blood cells, platelets and other constituents included in the plasma. We will discuss binary fluids later. In the case of low concentrated suspensions, that we will briefly describe here (indeed the higher concentration case is dealt with in the next section on particulate gels), the main characteristics are:

- Zero-shear viscosity determined by Einstein (1906, 1911) and improved by Batchelor (1977) after including the effect of Brownian motion in the case of spherical particles: $\eta=\eta_{s}\left(1+2.5 \phi+6.2 \phi^{2}\right)$ where $\eta_{s}$ is the solvent's viscosity and $\phi$ the volume concentration of particles.
- Shear-thinning effect: viscosity decreasing with shear rate, more pronounced as $\phi$ increases. Sometimes shear-thickening effect (viscosity increasing with shear rate).

- Yield stress at higher particle concentration (see next part on particulate gels).

- Non-vanishing normal stress differences.

- Effect of shapes and sizes of particles (aspect ratio $p$ ).

When particles are not spherical, $p$, the aspect ratio, is defined to be the ratio of the length over width perpendicular to the axis. $p$ can be greater than 1 (prolate spheroids) or smaller than 1 (oblate spheroids). One can show that the unit vector $\boldsymbol{u}$ parallel to the axis of symmetry of the particle is the solution of Jeffery's orbit (Jeffery, 1922):

$$
\dot{\mathbf{u}}=\mathbf{u} \cdot \mathbf{\omega}+\lambda(\mathbf{u} \cdot \mathbf{D}-\mathbf{u u u}: \mathbf{D})
$$

where $\lambda=\left(p^{2}-1\right) /\left(p^{2}+1\right)$ and $\mathbf{D}$ is the usual symmetric part of the velocity gradient tensor.

This equation has solutions which give rise to the wellknown tumbling motion (encountered with red blood cells for instance), i.e. the particle (except an infinitely long ellipsoid) keeps rotating continuously in a shear flow with given periodicity.

In general, after solving Eq. (22), one can then construct a stress field, which contains averages of the directions $\mathbf{u}$ over the whole space. The most complete expression is given by Hinch and Leal (1972). Three contributions are proposed, the one from the solvent $\boldsymbol{\sigma}_{\mathrm{s}}=2 \eta_{\mathrm{s}} \mathbf{D}$, the one that accounts for Brownian motion $\boldsymbol{\sigma}_{\mathbf{b}}=3\left(p^{2}-1\right) /\left(p^{2}+1\right) \nu k_{B} T\langle\mathbf{u u}\rangle$, and finally the one computed from the contribution of the ellipsoidal particles $\boldsymbol{\sigma}_{\mathbf{v}}$, also called viscous stress:

$$
\begin{aligned}
\boldsymbol{\sigma}_{\mathbf{v}}= & 2 \eta_{\mathbf{s}} \phi\{A\langle\mathbf{u u u u}\rangle: \mathbf{D} \\
& +B(\langle\mathbf{u u}\rangle \cdot \mathbf{D}+\mathbf{D} \cdot\langle\mathbf{u u}\rangle)+C \mathbf{D}\}
\end{aligned}
$$

where $A, B$ and $C$ are constants depending on $p$, the particle aspect ratio. The double dot sign means the product of a fourth order tensor operating on a second order tensor, and the brackets \langle\rangle mean averaging over all possible directions of $\mathbf{u}$.

There are also studies concerned with the study of rigid rods in a solvent, which show strong anisotropic effects. Doi and Edwards (1986) studied the effect of such rods using their model and proposed solving a Smoluchowski equation for the probability of finding a rod with orientation $\mathbf{u}$, in the semi-dilute case. Finally they come up with different constitutive equations which retain the right feature for these suspensions.

\section{Gels}

There are different kinds of gels, and different classes of gel materials. These systems are interesting, as we will 
see, because the cell cytoplasm may be regarded as a gel. Among gels, one finds polymer gels and particulate gels (Larson, 1999). A gel is a system, which is such that there are links between the micro-domains, which are present throughout the sample. These links may be weak or strong, depending on the kind of interactions involved. One may call gels physical or chemical gels. In a physical gel, the microstructure can be changed or released and the system can flow above some critical stress (Yield stress); then it can reform physical links when at rest. In a chemical gel, bonds are stronger, and they need to be broken so that the system can flow. The difference is that they will not form again afterwards.

\section{Polymer Gels}

These are made of polymers included in a solvent, which can be added and account for gel swelling. These polymers form bonds or links between them. As the concentration of bonds $(p)$ is increased, it reaches a critical one $\left(p_{c}\right)$, which corresponds to percolation. After this concentration has been reached, the gel will behave more like an elastic material, as illustrated in Eqs. (18) and (19). When studying dynamic properties of such gels (Winter and Chambon, 1986), it was found that, near the critical transition ( $p \approx p_{c}$ ), special properties with special exponents are obtained. If one defines a shear elastic modulus it can be shown to vary as $G \approx G_{0}\left(p-p_{c}\right)^{\mathrm{t}}$. The dynamic moduli $G^{\prime}$ and $G^{\prime \prime}$ behave roughly in the same way with a typical exponent $n$, so that $G^{\prime} \approx A \omega^{n}$, where $n$ is close to 0.6 (Winter and Chambon, 1986; Schmidt et al., 2000a). Depending on the type of polymers used (polydimethylsiloxane, polybutadiene, telechelic polymers, etc.), gels can undergo phase transitions, governed by the changes in the microstructure of the systems. In some cases, they might even give rise to some shear thickening. But in general, the Yield stress is a typical important parameter and it can be related to the self-similar structure or fractal of the system, i.e. some typical power of the concentration $p$ (de Gennes, 1979; Guenet, 1992).

\section{Particulate Gels}

These gels are formed when the concentration in a suspension of particles becomes large. At the level of concentrations used, the particles interacting with each

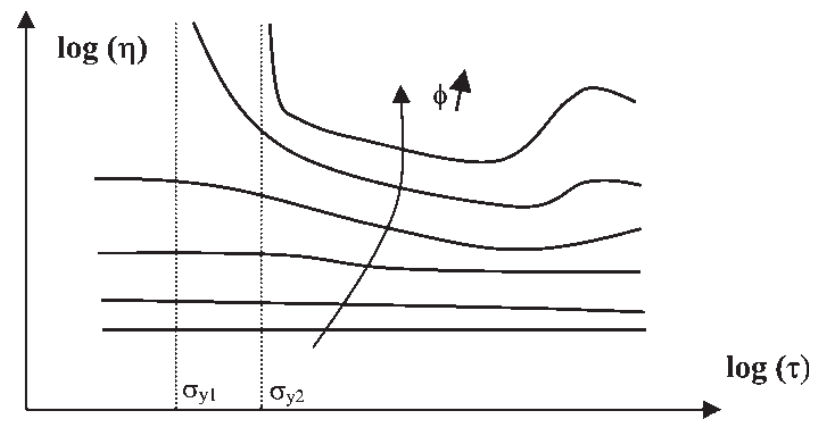

FIGURE 6 Viscosity (Pa.s) vs. shear stress $\tau$ (Pa) for a suspension of volume concentration $\phi$, redrawn from Laun (1984). other tend to form spatial structures; these structures are responsible for the formation of a network, associated with a Yield stress. Figure 6 displays the flow curves of a typical suspension of poly(styrene-ethylacrylate) particles in water at different concentrations (Laun, 1984). As the concentration is increased, the system shows evidence of a Yield stress (where $\tau=\sigma_{12}$ is the shear stress) because the shear stress goes towards a limit $\sigma_{y}$ in a $\log -\log$ scale plot. Also, one can notice the shear thickening at the higher shear rates or shear stresses. The Yield stress is an increasing function of the concentration (Pignon et al., 1997), because the higher the concentration, the more closely packed the particles, and therefore the harder it is to shear the suspension (see respective positions of $\sigma_{y 1}$ and $\sigma_{y 2}$ as a function of the concentration $\phi$ ). Equations for describing such systems are more sophisticated than Eq. (15). They include a yield stress condition, as in Eqs. (18) and (19). When dealing with models related to suspensions, special attention is needed regarding the particle-particle interacting potential, which forms the basis of the interactions, models, and relevant microstructures obtained. Dynamic measurements have also revealed plateaux for the $G^{\prime}$ and $G^{\prime \prime}$ moduli at the low frequencies, because in such cases, the system does not flow, as was shown in Fig. 3. This means that these suspensions behave as viscoplastic materials.

\section{Binary Systems}

Binary systems vary and can range from a fluid-fluid system to more concentrated ones where phases can coexist in a complex manner or architecture. Their names are emulsions, foams, blends, self-assembling fluids, etc. There are various theories which cannot all be listed here but can be referred to when dealing with such systems:

- small concentrations: laws for semi-dilute suspensions can apply;

- semi-dilute: Oldroyd model for Newtonian emulsions (Oldroyd, 1953, 1955), model for viscoelastic emulsions (Palierne, 1990);

- nonlinear transient behavior of concentrated polymer emulsions (Doi and Ohta, 1991).

In the case of two-phase fluid systems, the phenomena governing the dynamics of the system are coalescence (Verdier, 2001) and breakup (Grace, 1982) of droplets, which govern the rheology of the mixture. As the concentration is increased, the microstructure becomes more interesting and can go from droplets to cylinders or even to sheets (see for example polymer-polymer systems including copolymers). But the most interesting case is the one where one phase is present in small amounts but manages to form smart structures with poles and rods, as in the case of some polymeric systems or dry foams (Weaire and Hutzler, 1999). Such systems can flow but 


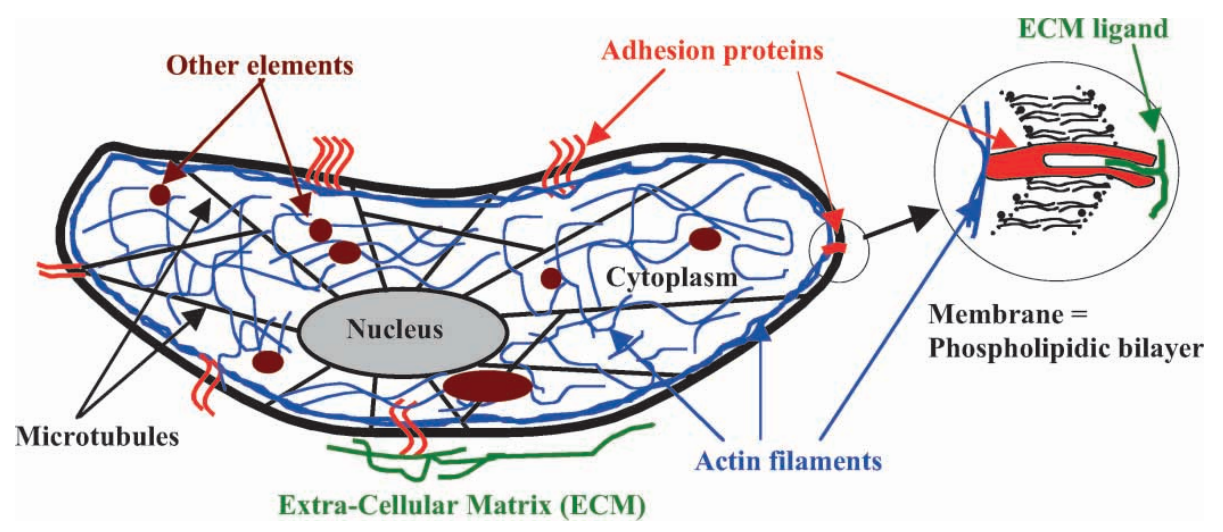

FIGURE 7 Sketch of a eucaryote cell.

also exhibit yield stresses. They can be considered to exhibit a cellular architecture, from a geometrical and mechanical point of view.

Finally another important case is that concerning micellar solutions where hydrophilic and hydrophobic components are present. Such systems lead to segregation of the hydrophilic parts on one side, and the hydrophobic on the other. The structures, which are formed, are very important; they include spherical micelles, cylinders, bilayers (membranes), planar bilayers and finally inverted micelles (Israelachvili, 1992). These systems can be investigated using theories based on chemical potentials, i.e. free energies (Safran, 1994; Lipowsky and Sackmann, 1995). In fact, these theories are also valid in general for the study of two-phase systems. Micellar solutions such as surfactants are important in everyday life. However, bilayers such as membranes are the major constituent of vesicles and cells (phospholipid membranes) and have been studied extensively. Their rheological properties are not so well known.

\section{RHEOLOGY OF THE CELL}

In order to understand how the rheological properties can be associated with the elements contained within the cell, we first describe what a cell is, what it is made of, and how the different elements can be compared to the materials previously described.

\section{Biological Description of the Cell}

Let us start first with a sketch of the components inside a cell. Figure 7 shows a typical eucaryote cell; such a picture can be found in the literature (Alberts et al., 1994; Humphrey, 2003) and therefore we refer to these more accurate books on cell biochemistry for a precise definition or more complete understanding of such systems.

We will now discuss the different elements contained inside the cell, as well as the membrane, the extra-cellular matrix (ECM), to see what kind of model, if any exists, can best describe an individual cell.

\section{Cell Cytoplasm-Nucleus}

The cytoplasm is a very complex system involving various objects present on different scales. The nucleus contains the genetic information and is composed of long DNA chains. These form two helicoidal chains wrapped around each other and can be very long. The nucleus is rather dense and behaves in an elastic manner. It is depicted as an ellipsoid in Fig. 7. An important biochemical aspect is the transduction of signals, which come from the membrane or other parts of the cell and arrive at the nucleus. This information is then recognized, and the machinery can start. The DNA is duplicated into RNA and then new kinds of polymers (i.e. proteins) are synthesized which will stay inside the cytoplasm or migrate to the surface of the cell, i.e. the membrane.

From the nucleus starts a network of filaments (cytoskeleton) which continues towards the membrane. Several types of filaments can coexist: microtubules, actin filaments, and intermediate filaments (Alberts et al., 1994). All these filaments are quite important and give the cell a rigid structure, even at equilibrium. This structure exhibits pre-stresses and will change its internal organization as the cell moves. During cell migration, for example, it is well known that the actin complex (in association with myosin) reorganizes itself to form a more rigid pattern of closely aligned actin filaments $(20 \mathrm{~nm})$ at the front of the cell. Then the cell can pull onto cell adhesion molecules (integrins for example), which are anchored to the cytoskeleton on one side and to the ECM on the other side (exterior of the cell membrane). In the middle of the cell (region of the cytoplasm located between the nucleus and the membrane), loose bundles of actin filaments, and regions similar to gels, have been observed. Therefore, the actin filaments are located in the various parts of the cytoplasm; they have a constant concentration and are more concentrated close to the membranes, where they form the cortical structure 
(see Fig. 7). They need to reorganize fast enough when cell migration is initiated. Their organization has been studied and seems to be well understood now, thanks to the new fluorescence techniques available nowadays. In the case of microtubules and intermediate filaments, the organization is far more mysterious and has not been studied so intensively. Basically, microtubules form long poles, which can be attached close to the nucleus and also at the membrane. All these filaments are quite important and form the basic idea of tensegrity models (Ingber and Jamieson, 1982; Ingber, 1993) which will be described in the next part.

The cytoplasm also contains other biological structures, in particular, mitochondria (energy exchange), vesicles (transport of proteins), and other large structures (endoplasmic reticulum, ribosomes and the Golgi apparatus) which can be deformable entities. These entities do not have a very active role in terms of cell deformation, but they do have a very fundamental biological action during the cell cycle.

To summarize, we may say, if we ignore the small scales involved due to the presence of small components, that the cytoplasm basically resembles a gel filled with more or less rigid particles (micron size). Its rheology should be associated with the general gel properties described before.

\section{The Cell Membrane}

The cell membrane plays a fundamental role in the cell life. Let us summarize its main functions. It needs to allow or prevent diffusion (water, solutions and ions). Also it is a very flexible structure ( = lipid bilayer, typical thickness $10 \mathrm{~nm}$ ) with a defined curvature, allowing for cell deformations, such as:

- formation of protrusions during migration (Condeelis, 1993);

- cell transmigration (diapedesis, metastasis) (ChotardGhodsnia et al., 2003);

- cell division (He and Dembo, 1997).

In all these situations, the cell and the membrane need to be highly elastic, deformable objects but also are in close association with the cytoskeleton (in particular the underlying actin network), so it is hard to define what is the actual responsibility of each one. Basically, a lipid bilayer has been shown to exhibit an elastic free energy of the form (Helfrich, 1973; Safran, 1994):

$$
f=2 k\left(c-c_{0}\right)^{2}+k^{\prime} c_{g}
$$

where $k$ and $k^{\prime}$ are two constants related to the elasticity of the membrane, and $c$ and $c_{g}$ are the main curvatures, that is to say the mean curvature $c=1 / 2\left(R_{1}^{-1}+R_{2}^{-1}\right)$ and the Gaussian curvature $c_{g}=\left(R_{1} R_{2}\right)^{-1}$. In particular, the constant $k$ is equal to $E h^{3} / 12 /\left(1-\nu^{2}\right)$ (Landau and Lifshitz, 1959), where $h$ is the membrane thickness,
$E$ the Young modulus and $\nu$ the Poisson ratio. With this in mind, it is possible, in a given situation, to determine the shape of a membrane in equilibrium (or during motion). It is also possible to determine changes in the effective elasticity moduli when one introduces proteins at a certain concentration (Divet et al., 2002).

Therefore, membranes are considered to be elastic (Landau and Lifshitz, 1959), but at higher levels of stresses, they will behave as nonlinear elastic sheets. Other models have been proposed (Skalak, 1973; Skalak et al., 1973; Evans and Skalak, 1980) using strain energy functions such as those in Eq. (13), but where the moduli and stresses are two-dimensional, like line tensions $(\mathrm{N} / \mathrm{m})$. These models work relatively well for describing the nonlinear properties of a red blood cell (Skalak, 1973).

The other main function of the membrane is to regulate the interactions of the cell with its environment, that is the neighboring cells and the ECM (see Fig. 7). This role is of major importance when a cell starts its motion and needs to show the correct affinity with the ECM (Palecek et al., 1997), in other words, not too strong and not too small. Also cell-cell interactions are essential for maintaining the correct adhesiveness between cells so that tissue integrity is preserved. Through all these interactions, the connection between the binding proteins (called CAMs, Cell Adhesion Molecules) with the ECM or the cell cytoskeleton (actin network) is sometimes needed, as depicted in Fig. 7. Some CAMs are indeed transmembrane proteins and can attach the cytoskeleton in a rigid manner. On the other end, they form, like the integrins, a "binding pocket" into which other molecules or ECM constituents (collagen, elastin, polysaccharides, laminin, fibronectin, etc.) can fit and bind efficiently.

Finally, cells sense their environment by precisely using adhesion molecules or other small molecules (Leyrat et al., 2003) to determine in what direction they want to go. Then such molecules are able to generate signaling cascades, which end at the nucleus, and to the possible creation of new CAMs, or to other events. This can give rise to reinforcement of the attachment of the cell with another one (or with the ECM, see below), or conversely to the breaking of bonds, thus allowing migration.

\section{The Extra-Cellular Matrix (ECM)}

Extra-cellular components are generally needed for the connection between cells. The main ones are collagen, elastin, polysaccharides, fibronectin, laminin, etc. They are generally made of polymer chains or long filaments, which are interconnected with each other and have structures close to gels. Enzymes can degrade these gels when cells are migrating and produce such entities to degrade this filamentous structure. There is a possibility nowadays to construct model tissues using collagen gels, where real cells are embedded.

The components of the ECM are important because they form the basis of the ground where cells 
adhere and through which they migrate. When a cell is simply put onto a glass plate, if there is no adherence or ECM components, then the cell will make them on its own. For example, a human umbilical vascular endothelial cell (HUVEC), which is a cell constituting the vascular walls in a vessel, is able to make its own fibronectin, which is useful for it to adhere firmly onto glass. Fibronectin is indeed a ligand of the integrins ( $\alpha \beta$ structure) which can form binding pockets (Alberts et al., 1994), such as that depicted in Fig. 7. The two branches of the integrin ( $\alpha$ and $\beta$ ) can change their conformation to allow a specific ligand to enter and adhere through the presence of multiple weak interactions. This mechanism is also possible in the case of the adhesion of integrins with other adhesion molecules (heterophilic bonds), such as the immunoglobulin family. Due to the gel structure of the ECM, the cell can pull strongly on these bonds (e.g. integrin-fibronectin) and is able to migrate.

\section{The Cellular Object}

Finally, to summarize the description of a cell, we may possibly depict it as a "bag" containing a complex fluid. This complex fluid may be modeled as a viscoplastic material, as long as we can ignore the nucleus. Indeed, measurements of F-actin solutions, for example (Schmidt et al., 2000a), have revealed that the low frequency behavior of the dynamic moduli $G^{\prime}$ and $G^{\prime \prime}$ is as shown in Fig. 3, in other words close to a plateau. This has also been observed on human airway smooth muscle (HASM) cells (Fabry et al., 2001), where the moduli dependence is similar. Such examples will follow in the next part. In general, it is also necessary, when possible, to include a rigid elastic nucleus, as in the modeling of leukocytes during flow (Tran-Son-Tay et al., 1998) or even better, to add a viscoelastic nucleus (Verdier et al., 2003). With the latter method, it is possible to follow the cell deformation when a cell is adhering and then is spreading onto a surface.

A cell is then depicted as a complex object (viscoplastic fluid) containing a viscoelastic nucleus, the whole composite medium being surrounded by a membrane. Of course, this description does not take into account any biochemistry or signaling. Indeed, one would need to add a "live" parameter (or more) which could monitor changes in the organization of the fluid's elements. Such a description is rare, but one can refer to Dembo's work (He and Dembo, 1997), which predicted cell division, using a non-constant (or non homogeneous) viscosity: this viscosity is defined as a function of the actin concentration. The actin concentration rules the viscosity similarly to a sol-gel transition and allows for a time- and space dependent-viscosity through the evolution equation of this parameter. Such models are often found in the literature when dealing with thixotropic systems, which are materials with the ability to change their structure when applying different stresses or forces, like in flow situations, for example.
In any case, this is just an attempt to describe best how the cell could be modeled, because it is such a complex object that there is no ideal law to describe it.

\section{Microrheology at the Cell Level}

Let us try to describe now how experiments can be carried out at the cell level. We will call this subject microrheology, because it is the name given nowadays, by contrast with the conventional rheometrical techniques developed in the past. In fact, the best name to be used should be microrheometry. During the past decade, recent advances have been made thanks to the efforts achieved by biophysicists and due to the combination of techniques coming both from physics and biology. The first important idea to be developed is what do we want to measure, and what can we really measure at the cell level?

\section{Length Scales}

Most of the techniques used nowadays are interested in testing the cell on a small scale, say the subcellular level. In theory, this sounds like a nice idea, but in practice it is sometimes not possible. Classical continuum mechanics theory (Sedov, 1975; Fung, 1993b) claims that, for measuring a certain macroscopic parameter, the size of the sample considered for the test should be much larger than the size of a typical subunit in the system (Batchelor, 1967), say fifty times larger. Referring to Fig. 7, we can foresee that parts of the cellular cytoplasm may be tested as a whole, but that some parts might not, because the present are the objects too large. This of course depends on the size of the probe used. We will see in the next part that probes are usually microspheres, microneedles, micronic objects. Considering this aspect, we may conclude that probing the nucleus with a micron-size sphere is something possible in terms of size, as well as regions of the cytoplasm containing networks of actin solutions, and also the membrane. One must remember that in the latter case, the membrane can be tested but its response will be significant in terms of what is also lying underneath, including the cytoplasm. A nice piece of work is the one by the group of Sackmann (Schmidt et al., 2000a), where both microrheological properties (using magnetic tweezers) and macrorheological ones are carried out. They found that microrheology underestimates the $G^{\prime}$ and $G^{\prime \prime}$ moduli measurements, in the case of F-actin solutions representing the cytoskeleton. In a second paper (Schmidt et al., 2000b), the same group showed that by using the same techniques, they were able to obtain some agreement between micro and macro data. This is because the probe $(4.5 \mu \mathrm{m})$ is large compared to the subunits studied, unlike in the previous case (Schmidt et al., 2000a).

The last problem to test the cell is still to find a way to insert a probe into the cell. Indeed the cell will always attempt to engulf the object or probe. In active microrheology methods, this needs to be achieved first. 
Other methods, based on the direct observation of the Brownian motion of an object (vesicle for instance) sound more promising. Finally methods attempting to investigate the cell membrane may be simpler, because the probe does not need to be inserted; indeed the probe needs to be attached to the cell membrane, which is more common. But in such cases, one measures the response of the membrane and the underlying cytoskeleton (actin here), so the interpretation is more intricate. Let us now review the different possible ways to determine the microrheological properties of an individual cell.

\section{Physical Methods to Investigate Cell Microrheology}

The methods proposed here are not exhaustive, but we present those which have led to significant advances in the field of microrheology. Note that these techniques are all based on theories, which use various assumptions that will also be explained. Assumptions rely on a cell model, but are also based on a certain way to analyze the data.

\section{MicropipetTES}

Micropipettes have been developed both theoretically (Yeung and Evans, 1989) and experimentally (Evans, 1973; Evans and Yeung, 1989). The basic idea is to draw cells (blood granulocytes in their case) into a smallcalibrated micropipette (diameter around $2-8 \mu \mathrm{m}$ ) at different suction pressures $\left(0.1-10^{5} \mathrm{~Pa}\right)$, corresponding to a wide range of forces $(10 \mathrm{pN}-10 \mu \mathrm{N})$. Cell areas can be extended to twice their initial value. The model (Yeung and Evans, 1989) assumes that the cell is made of a cortical layer surrounding a viscous fluid, and calculates numerically the flow induced by a constant pressure onto the model cell. Therefore, the apparent viscosity and the cortical tension may be deduced. Typical values of the suction pressures are $10^{2}$ to $10^{4} \mathrm{dyn} / \mathrm{cm}^{2}$. The associated viscosities range between $10^{3}$ and $10^{4} \mathrm{P}$, depending on the temperature. Finally, cortical tensions are around $0.03 \mathrm{dyn} / \mathrm{cm}$, and do not affect the results so much. The results depend on the pipette-to-cell-size ratio and on the ratio of viscous effects divided by cortical stresses. Other interpretations of such experiments can also provide access to shear rigidity moduli (Evans, 1973), around $6 \mu \mathrm{N} / \mathrm{m}$.

This method has been modified to determine adhesion energies (Hochmuth and Marcus, 2002) between the lipid bilayer and the underlying skeleton in the case of neutrophils and RBCs, and they also measure effective viscosities. In a review paper (Hochmuth, 2000), it is shown that the viscosity of neutrophils is in the range of $100 \mathrm{Pas}$, and their cortical tension is about $30 \mathrm{pN} / \mu \mathrm{m}$, whereas chondrocytes and endothelial cells behave as viscoelastic solids with an elastic modulus around $500 \mathrm{~Pa}$. Also the method can be used as a force-measuring system, using RBCs as springs, combining the use of two micropipettes, and has been adapted for the measurements of interaction forces.

\section{AFM}

The AFM apparatus is based on the scanning tunneling microscope and has been improved to become what it is nowadays (Binnig et al., 1986). It is based on the following principle: a cantilever with a sharp edge is brought into close contact with a surface or object, and forces can be exerted onto it. The cantilever deflection provides access to the force $(10 \mathrm{pN}-100 \mathrm{nN})$ by use of a laser falling onto the cantilever. The angle change is related to the deflection and, therefore, to the force, once the system is calibrated. Micro or nanodisplacements are usually possible through the use of piezotransducers, which allow very good position accuracy. Usually the sample is lowered to come into contact with (or close to) the cantilever. AFMs are particularly well adapted for measuring adhesion forces and microrheological properties of soft biological objects, like cells. Different techniques can be used to measure forces, (a) contact mode, (b) non-contact mode, and (c) tapping mode. The latter two are interesting because they allow samples to be tested without contact. The contact mode is also interesting because it allows cell to be indented or stretched (Canetta et al., 2003). Force dynamic spectroscopy (FDS) is made possible and allows measurement of time-dependent forces, in the case of adhesion forces (Canetta, 2004). Different cone tips can be used with different angles and shapes; usually the size of the radius of curvature is about $20 \mathrm{~nm}$.

The first application of AFM for cellular systems has been to establish elastic mappings of living cells. Using Hertz elastic theory (Hertz, 1896) of indentation, and a scanning probe AFM, it was possible to draw elastic mappings of canine kidney cells (A-Hassan et al., 1998). This method has been improved recently (Canetta et al., $2003)$ to determine both the local elastic modulus $(E)$ and adhesion energy $(\gamma)$ of Chinese Hamster Ovary cells (CHO), using the JKR test (Johnson et al., 1971). The latter uses the following relation:

$$
a^{3}=\frac{R}{K}\left[F+3 \pi R \gamma+\sqrt{6 \pi R \gamma F+(3 \pi R \gamma)^{2}}\right]
$$

where $a$ is the cell-microsphere contact radius, $R=R_{1} R_{2} /\left(R_{1}+R_{2}\right)$, with $R_{1}$ being the cell radius of curvature, and $R_{2}$ the radius of the bead (typically $15 \mu \mathrm{m}$ ), $F$ is the applied force and $K=16 E / 9$ for incompressible systems. A typical indentation test is shown in Fig. 8a, with a view from the side. Typical values for the moduli are $0.2-0.8 \mathrm{kPa}$ and adhesion energies $0.4-4.10^{-5} \mathrm{dyn} / \mathrm{cm}$, depending on the proteins present on the cell and bead.

Further tests have been carried out in which a cell is stretched (Fig. 8b), thus enabling the nonlinear timedependent response of a cell (with adhesion peaks superposed) to be determined, and this can be predicted using Eqs. (11-13), for example. These experiments have also been carried out by other authors (Wojcikiewicz et al., 2003) using leukocytes, who show that the adhesion interactions can also affect the mechanical properties, as also shown by our group (Canetta, 2004). 

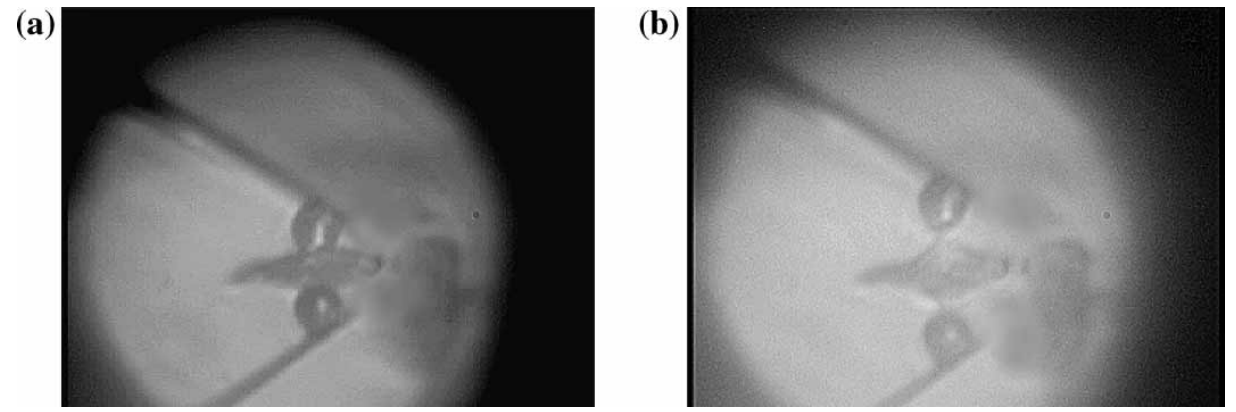

FIGURE 8 (a) Indentation of a CHO cell, seen from the side (with reflection), $R_{2}=15 \mu \mathrm{m}$. (b) Stretching test with tether formation (CHO cell), $v=1 \mu \mathrm{m} / \mathrm{s}$ (Canetta, 2004).

Another interesting experiment based on a generalized Hertz model (Mahaffy et al., 2000) gives frequencydependent moduli of polymer gels and biological cells. This demonstrates that cells have a behavior close to gels and exhibit plateau moduli, which are almost frequencyindependent.

\section{Microplates}

We now describe a recent technique (Thoumine and Ott, 1997) derived from the study of the AFM, except that the cantilever is a microplate, which is set perpendicular to the microscope. In this set-up, one can visualize (from the side) a cell which is located in between two microplates, one of them being the cantilever, or force transducer. The cell can be compressed or pulled. It is the deflection, which gives access to the force, after calibrating. Interesting videos from the side exhibit the cell as time moves on. Typical forces are in the $\mathrm{nN}$ range (around $10^{-8} \mathrm{~N}$ usually according to Thoumine and Ott (1997)). Nonlinear deformation of the cell is obtained (strain of about 2) until the cell is detached from the plates. 1D-viscoelastic constitutive equations have been used to predict the cell deformation, but actually a nonlinear model such as those for nonlinear elastic materials would be more convenient.

A similar approach (Caille et al., 2002) has been used in the case of endothelial cells. Particular attention is paid to the role of the nucleus during deformation. The nucleus and the cytoplasm have been described as hyperelastic materials, Eq. (12), and compression tests were carried out. Simulations give access to the elastic moduli of the cytoplasm $(500 \mathrm{~Pa})$ and of the nucleus $(5000 \mathrm{~Pa})$ and constitute an interesting way to have access to such properties.

\section{Optical TweEzers}

Optical tweezers have been discovered about fifteen years ago (Ashkin and Dziedzic, 1989; Sheetz, 1998), and they use the principle of the power of a laser source (around $300 \mathrm{~mW}$ ) focused into the objective of a microscope so that a particle or cell submitted to the light intensity becomes trapped. Once the particle moves out of the trap, a restoring known force can be measured (in the $\mathrm{pN}$ range, say from 0 to $200 \mathrm{pN}$ ) after calibration.
Such systems are now quite common and have been used for applying forces to cells while measuring their deformation. The technique can be improved when adding systems capable of moving the laser beam fast, such as acousto-optic modulators (Helfer et al., 2001). With this technique, it is possible to catch several objects (beads or cells) one after the other, or to move two beads inside a cell quite rapidly. Such beads (micron size) need to be inserted using well-adapted protocols. As an example, small controlled forces can be applied to erythrocytes (RBCs, Hénon et al., 1999) to measure their membrane elastic modulus, which is about $2.5 \mu \mathrm{N} / \mathrm{m}$. This is slightly smaller than that calculated in micropipette experiments (Evans, 1973), i.e. around $6 \mu \mathrm{N} / \mathrm{m}$. These discrepancies will be discussed in the final part of this section.

More recently, optical tweezers have been combined with Particle Tracking experiments (Helfer et al., 2001) to lead to the analysis of the back-scattered field by the trapped bead. This intensity can be used to gain access to the complex dynamic modulus of the system, and has been used with polymer solutions (F-actin biopolymer solutions, polyacrylamide gels) within which beads are embedded (Gittes et al., 1997). This made it possible to analyze the properties of actin-coated membranes after attaching beads to them. The presence of the actin network seems to modify the membrane dynamics as expected. A 2D-membrane shear modulus (around $5 \mu \mathrm{N} / \mathrm{m}$ ) has also been reported, and a viscoelastic character is finally found for the membrane. Finally, a recent book on the subject (Sheetz, 1998) is now available and contains more detailed information about optical (or laser) tweezers.

\section{Magnetic TweEzers}

This microrheology technique implies imbedding magnetic particles inside a sample to be tested and applying a magnetic field. These beads can also be located on the cell surface, if one wants to investigate the coupled properties of the membrane with the underlying cytoskeleton. Using videomicroscopy, one can determine the position and motion of the particles. The resulting displacements provide access to the microrheological response of the surrounding medium. In fact, the torque is measured through the applied magnetic field, 
and the displacement gives the angle, associated with shear deformation. The magnetic fields required are not so large, because particles are rather small (typically $1 \mu \mathrm{m}$ ), even though a large number of them can be required.

Such a technique has been proved to be efficient for measuring the properties of actin networks (Schmidt et al., 1996; 2000a) and filamentous bacteriophage fg (Schmidt et al., 2000b), but also in real cells such as macrophages (Bausch et al., 1999), using creep data. This allowed the authors to determine the viscosities and elastic moduli, as modeled by a Kelvin model. The moduli are about $350 \mathrm{~Pa}$ whereas the viscosity of the cytoplasm is $210 \mathrm{~Pa}$. These values are somewhat different from those from the literature. Another study (Fabry et al., 2001) using larger beads $(4.5 \mu \mathrm{m})$ shows that there is a scaling law, which governs the elasticity and viscosity coefficients. This means that soft biological systems can behave like glassy materials, close to the glass transition (Sollich et al., 1997; Sollich, 1998).

\section{Particle Tracking Microrheology}

So far the methods described were all active techniques. We will now describe another technique, which is a passive one, since it allows one to the motion of a particle or micronic system due to Brownian motion only, without applying any force or displacement. This technique leads to the measurements of the dynamic moduli in the range of a few hundred $\mathrm{Pa}$. The timedependent position correlation function or mean square distance (MSD) is:

$$
\left\langle\Delta \mathbf{x}^{2}(t)\right\rangle=\left\langle|\mathbf{x}(t+\tau)-\mathbf{x}(\tau)|^{2}\right\rangle_{\tau}
$$

where the brackets mean averaging over all times $\tau$, and $\mathbf{x}$ is the position of the center of mass. In the case of simple diffusion in a liquid, we have $\left\langle\Delta \mathbf{x}^{2}(t)\right\rangle=2 d D t$ ( $d$ is dimensionality), and the diffusion constant $D$ is related to the viscosity by $D=k_{\mathrm{B}} T / 6 \pi \eta a$ (Stokes-Einstein equation), where $a$ is the particle radius. In the case of viscoelastic materials, the formula can be generalized (Mason and Weitz, 1995; Gittes et al., 1997; Mason et al., 1997; Schnurr et al., 1997):

$$
\tilde{r}^{2}(s)=\frac{k_{\mathrm{B}} T}{\pi a s \tilde{G}(s)}
$$

where $s$ is the Laplace frequency, and tildes denote the Laplace transform (of the MSD or relaxation function). The information on both elasticity and viscosity is, therefore, contained in Eq. (27) and allows for the measurements of the microrheological properties, after transforming $G(s)$ into the Fourier domain, to obtain $G^{\prime}$ and $G^{\prime \prime}$. The only techniques required are to insert the bead into the system, and to visualize the bead movements. This is done usually using the light scattered by the particle. Two methods are commonly used, diffusing wave spectrometry (DWS) (Pine et al., 1988), which allows higher frequencies, or quasi-elastic light scattering
(QELS) (Berne and Pecora, 1976), to be measured. In the end, the frequency range covered is usually $10 \mathrm{~Hz}-$ $100 \mathrm{kHz}$ (Dasgupta et al., 2002).

Measurements on uncross-linked flexible polymers (polyethylene oxide solutions) show that all methods (DWS, QELS and even conventional rheometry) agree (Dasgupta et al., 2002). The F-actin solutions have also been investigated (Gittes et al., 1997; McGrath et al., 2000; Tseng and Wirtz, 2001) but also living cells (Yamada et al., 2000).

Finally, we mention a new technique based on twopoint microrheology (Crocker et al., 2000), which has been developed because a particle may damage its surrounding medium, so if one wants to determine bulk properties, the use of two particles is better. By measuring the cross-correlated motion of two tracer particles (distance 10-100 $\mu \mathrm{m}$ ), the local effects can be eliminated. Note that this technique will be difficult to apply to cells, since typical rheological properties might change within a few microns.

\section{DISCUSSION}

All the methods presented here are very attractive but one needs to be cautious. Most have been tested first using model polymer solutions or polymer gels. It might become difficult to look at a cellular system. Indeed the size of the elements is such that changes can occur very locally. Another related problem is the insertion of beads (since beads are used quite often) or other micronic objects inside the cell. This operation is difficult because the cell will encapsulate beads by forming a new lipid bilayer (phagocytosis). Then we are not sure what properties are actually measured. There is still some work to do regarding cells.

The discrepancies between measurements of the same parameters by different methods, operators, conditions or cells are of course a natural consequence. Indeed the rigidity of a fibroblast membrane will never be a universal parameter! To this we need to add the different assumptions made when interpreting the data, using models. Finally a single cell is always unique, and it is ruled by its own life cycle, which changes its properties sometimes drastically.

Finally, we may observe that the frequency dependence of the dynamic moduli $G^{\prime}$ and $G^{\prime \prime}$-as measured using particle tracking microrheology-is interesting, but is far from being satisfactory to conclude on a 3D model. Indeed, separate experiments are needed to provide access to all the parameters in the different models presented in the first part of this work. This is frequently done in conventional rheology. The techniques for measuring large deformations are of particular interest, but remain unexplored, except in a few cases. Finally, some experimental models already use a certain constitutive law, which is in fact to be determined. Actually, we are more interested in solving an inverse problem rather than 
finding a few simple parameters corresponding to a simple 1D-model. Therefore, the use of combined techniques to measure different parameters (shear, elongation) under different combined situations (transients, steady state) is really the best way to determine the correct constitutive equation, if it exists.

\section{FROM THE CELL TO THE TISSUE}

\section{Are Tissues just a Macroscopic Generalization of the Cell Microscopic Properties?}

The previous part gave some hints about how to determine microscopic properties, such as dynamic moduli, adhesion energies, cortical tensions, elastic moduli, yield stresses and other such parameters used in the models presented before. Now the question is whether these properties can simply be generalized to obtain the general constitutive equation of a tissue (muscle, vascular walls, epithelium, etc.) or a biological fluid (blood, synovial fluid, etc.). In other words, can the material be elastic if the cell is elastic? And if so, what will its elastic modulus or Poisson's ratio be? Part of the question needs to be answered after looking at the scales involved. Usually, when one wants to look at the final tissue, we need to forget about what can be seen locally. There is a scale separation, as long as the size of the sample $\left(L_{\mathrm{s}}\right)$ is large enough compared to the size of a cell-element $L_{\mathrm{c}}$ (Sanchez-Palencia, 1980), and there are good chances of finding an equivalent medium. The idea is slightly different from that mentioned in the introduction, because we are now looking at a typical cell-element, which can be larger than the basic cell unit (size $L_{\mathrm{e}}$ ). This cell-element should be chosen to contain all the information that we have from microrheology, in particular, for a cellular system, the cell type, shape, cytoplasm, nucleus, constituents, cell cycle, membrane, proteins in presence, ECM, cell-cell, cell-ECM interactions, etc. Of course all this information will not be available! In practice, hypotheses need to be made so that something computable can be obtained.

The main difficulty is that the cell is a composite system, made of a membrane surrounding a complex fluid. There are different methods available in the literature, which will now be reviewed. They have not been applied to many cases of biomaterials. Viscoelastic effects are in fact difficult to include. But we will see that they can provide interesting tools for the investigation of complex biological systems.

\section{Mathematical Methods}

Mathematical models are usually necessary to go from the microstructure to the constitutive equations. Perhaps the simplest case to start with is the method considered by Fung (1988), when looking at the properties of the lung tissue. The lung tissue (or lung parenchyma) is showing a periodic alveolar structure, where each sub-element is a tetrakaidekahedron. All these geometric sub-elements are periodically assembled. Each edge becomes the edge of the alveolar mouth, and the alveolar mouth forms alveolar ducts (ventilation). The alveolar mouths and ducts are made of collagen fibers and elastin. The microrheological properties of these fibers are supposed to be well known. The edge is called a cable, and the interstitial matter a membrane, which has a two-dimensional behavior. To determine the constitutive equation (with and without ducts), or to obtain the elastic macroscopic parameters, we consider:

- the equilibrium of three membranes at a vertex-no bending moments in the cables;

- the principle of virtual work is applied to a polyhedron.

This gives access to the bulk modulus $K$, when a uniform transpulmonary pressure $P$ is applied. The bulk modulus, when ducts are present, is given by:

$$
K=\frac{2}{3}\left(F_{1}-\frac{1}{2}+0.3\left(F_{2}-F_{1}\right)\right) P
$$

where $F_{1}$ and $F_{2}$ are, respectively, the coefficients of membrane extension ( $\dot{T} / T=F \dot{A} / A, T$ stress, $A$ area) in the hexagonal and rectangular faces. When fibers are collagen or elastin, the stress is, respectively, given for these fibers by Eqs. (29a,b)

$$
\begin{aligned}
& T=\left(T^{*}+\beta\right) e^{\alpha\left(\lambda-\lambda^{*}\right)}-\beta \\
& T=E(\lambda-1)
\end{aligned}
$$

where $\lambda$ is the stretch ratio relative to zero-state, $\lambda *$ another stretch value, $E$ is the Young modulus of elastin, and $T *, \alpha, \beta$ are constants. By looking at a statistical system, one can derive the total stress exerted on the side of a unit square of fiber-embedded membrane (including different orientations) and have access to $F_{1}$ and $F_{2}$. Finally $K$ can be computed and is found to be of the order of $2 \times 10^{4} \mathrm{dyn} / \mathrm{cm}^{2}$, similarly to that obtained for middleaged humans. Equation (28) has the advantage of being simple, and other methods are usually more sophisticated but retain these basic ideas.

\section{Homogenization}

Homogenization was introduced over twenty years ago (Sanchez-Palencia, 1980) and is based on the following formalism. Considering a typical sub-element of a system, one may take advantage of the separation of scales to introduce a small parameter $\varepsilon=L_{\mathrm{e}} / L_{\mathrm{s}}$ which can be used to expand the solution (stresses, strains, velocity fields) in powers of $\varepsilon$. The solution depends on the equations of the problem considered, but it is known that such methods have been used successfully for understanding flow through porous medium, conductivity problems, etc. In some cases, the periodicity of the domain may be used 
so that simplifications can be obtained. The method does not always lead to satisfactory results, and may be ill posed. Then the system cannot be homogenized. We present here a few examples of the application of such techniques to the study of tissues.

The first one deals with syncytial tissues ( Neu and Krassowska, 1993). These tissues include the myocardium, muscle fibers, gastric epithelial cells, and the eye lens, but the method may be adapted to nonsyncytial tissues such as the skeletal muscle or nerve bundles. The periodicity of the tissue is used in this case. These tissues are multicellular systems, made of cells surrounded by a membrane, interacting with each other, and the smallest structural element that is considered is a small number of cells, which will be called the unit cell. The particular problem here is to solve for the electric field inside and outside cells. These fields are solutions of the Laplace equation, together with boundary conditions. A small parameter $\varepsilon$ is introduced, based on physical microscopic and macroscopic lengths, arising from the problem. Asymptotic representations (Bensoussan et al., 1978) of the problem can be written. Then by integration and use of the divergence theorem equations for intra- and extra-cellular domains can be obtained. These are the macroscopic equations. Finally, partial differential equations (reaction-diffusion equations) for the averaged intra- and extra-cellular potentials can be obtained to derive the macroscopic conductivity tensors. This method is also compared with the bidomain model (Clerc, 1976) and this reveals the limitations of the homogenization technique in the case of surface problems, proximity of external sources or finally with strong, nonuniform or fast changing external fields. This method is interesting, but does not provide information about homogenized mechanical properties.

Another problem is the homogenization of honeycomb structures (Lee et al., 1996), which are important when looking at structures, but can also provide a model for describing metallic foams. These materials are supposed to be made of regularly organized cells, and might also give a good description of human tissues. Two types are considered: honeycomb structures with regular hexagonal cells and re-entrant honeycomb structures. The basic unit is either a rectangle or a square. The elements are beams, which are supposed to be elastic, and a good description can be found elsewhere (Gibson and Ashby, 1988), thanks to a beam analysis of a unit cell. The technique used here is the homogenization finite element method (FEM) technique. A weak form of the elasticity problem is written, combined with asymptotic expansions in terms of a small parameter. A homogenized elasticity tensor appears when solving the problem and gives access to the effective elastic modulus $E_{\mathrm{e}}$ and Poisson's ratio $\nu_{\mathrm{e}}$. The influence of the volume fraction of the elastic structure can then be analyzed, and this is in rather good agreement with the model of Gibson and Ashby (1988). The regular structure has increasing elastic moduli and decreasing Poisson's ratio when the volume fraction increases. The re-entrant structure exhibits a decreasing
Poisson's ratio with increasing volume fraction and shows negative values depending on the angles of the structure. Note that a similar technique is used for the optimization of the design of periodic linear elastic microstructures (Neves et al., 2000). The problem is to find the optimal representative microstructural element which maximizes the equivalent strain energy density function or a linear combination of the equivalent mechanical properties.

We may conclude, therefore, that this model may be adapted to the study of tissues containing regularly spaced cells, with a given shape, but the influence of the cytoplasm and membrane would need to be included.

The final method presented here is a different one, called the discrete homogenization method (Tollenaere and Caillerie, 1998; Caillerie et al., 2003). It has been successfully applied to the homogenization of the myocardium. Cardiomyocites can be assumed to form a quasiperiodic discrete lattice, made of bars linked with each other's. They are supposed to be elastic and interact at nodes where moments are accounted for as an improvement of the work of Fung (1988) presented above. Balances of forces and moments are written out at the nodes, but a weak formulation (virtual power formulation) is preferred. The elasticity of the bars is introduced and can give rise to large deformations. Then the node's positions are expanded in terms of a small parameter (ratio of microscopic to macroscopic lengths), as well as forces and moments. Finally, an equivalent continuous medium is obtained, and a formula for the equivalent Cauchy stress tensor is found, when the number of elementary cells is large. Frame-invariant constitutive laws are found to be nonlinear. The equivalent medium is hyperelastic. Finally the method could be improved to include the effect of the ECM. Comparisons with actual experiments are unfortunately not presented, but the trends seem to be in agreement, because hyperelasticity of soft tissues is often found, as will be seen in the final part.

\section{Tensegrity}

The tensegrity concept (Fuller, 1961) is an idea for describing deformable structures made of sticks and strings under tension or compression. This model has been shown to be particularly well adapted for the description of the cell (Ingber et al., 1981; Ingber and Jamieson, 1982). But it has also been used in different domains like civil engineering in particular. The basic rules (Ingber, 1993) for this structure can be defined as follows:

- It is made of struts and strings that have a certain initial shape thus enabling pre-stressed states. Indeed a suspended cell has a usually spherical shape and is pre-stressed.

- When it is pressed onto a substrate, it flattens and spreads onto it. 


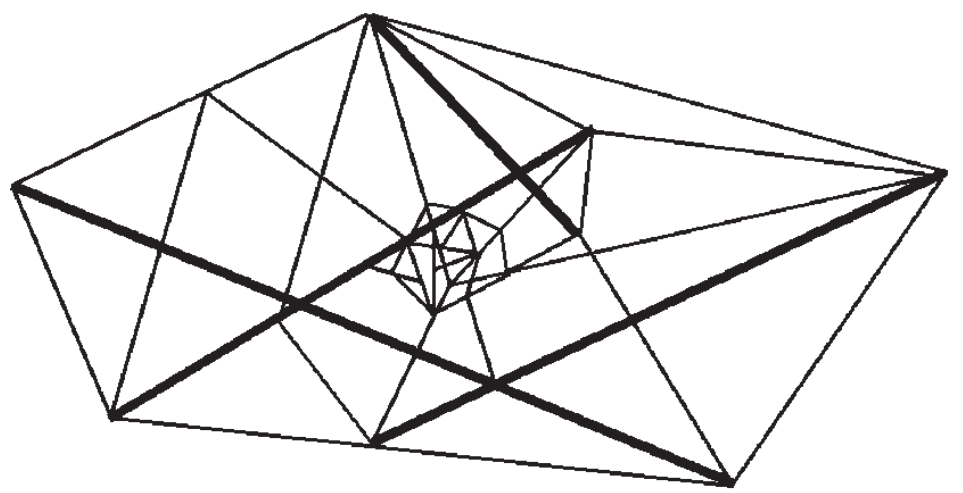

FIGURE 9 Tensegrity structure similar to a cell with nucleus (redrawn from Ingber, 1993).

- If the pressure is removed, it spontaneously jumps back, revealing elasticity.

Ingber et al. (1981) consider that cells, at first sight, might behave as tensegrity structures. The elements are able to sustain tension or compression forces. To determine global reactions, like stresses, or elastic responses of the tensegrity structure, one needs to apply the usual equilibrium equations on the number of elements and finally solve this numerically, because usually the number of elements can be large. Different sophisticated assumptions can be made for the sticks or cables, like nonlinear elastic elements (Wendling et al., 1999; 2000), or even viscoelastic cables (Canadas et al., 2002). A typical tensegrity structure representing a cell with its nucleus is shown in Fig. 9.

The elements are mainly large sticks and are connected by elastic wires. In the middle of the figure, the cell nucleus has also been represented using the same elements but they could also be different thin sticks linked with other types of wires. The physical nature of these sticks is closely associated with the cytoskeleton, in particular the different types of filaments present inside the cells, as mentioned previously, actomyosin complexes which form rigid polymer rods, microtubules, and intermediate filaments. By saying that, we understand that the model will show how the cell spreads, for example. It is not just one part of the cell cytoplasm which is responsible for deformations, but it is the cell as a whole structure which is set in motion. These arguments have raised an interesting debate, which is still being discussed (Ingber et al., 2000), as it seems difficult to understand that local phenomena cannot have stronger effects. The mechanical stresses which are applied to a cell need to be modeled in terms of mechanotransduction, i.e. how stresses generate other types of reaction within the cell, through signaling. Magnetic twisting devices have been used (Wang et al., 1993) to show that focal adhesion changes were induced as a result of these applied forces. This induced a force stiffening process, i.e. the cytoskeletal stiffness was found to increase linearly with stress (stress hardening). This was verified using tensegrity models, which were able to show this behavior, due to the use of interdependent struts.
This helped to understand the way mechanotransduction acts through rearrangements of the tensionally linked cytoskeleton. Finally, a more refined analysis (Wang et al., 2001), including microscopic observations revealed recently that cells behave like discrete structures composed of an interconnected network of actin filaments and microtubules. Microtubules seem to be responsible for the compression forces and determine cell-shape stability, i.e. initial stresses $\left(10^{2}\right.$ to $\left.10^{3} \mathrm{~Pa}\right)$. Dynamic and static tests seem to confirm the ability of the tensegrity model to predict the cell mechanical response.

To conclude, this model has the advantage that it starts from basic measurable elements and is in reasonable agreement with experiments.

\section{Cell Sorting: The Surface Tension Effect}

The concept of cell sorting has been proposed by Steinberg (1993) and is not necessarily a model which has its place here. Nevertheless, it is important to mention it as it forms the basis for future modeling where interfacial effects are present. In his early work, Steinberg compares the behavior of immiscible liquids to that of embryonic tissues. He remarks that the motions of spreading and rearranging of droplets in a surrounding medium are comparable to those of different cells put together, which are being sorted out, as in the case of morphogenesis. This could be explained by tissue interfacial tension $(\gamma)$ arising from cell adhesion. The only remaining problem is to prove the existence of an interfacial tension for cells. This idea has been confirmed later (Foty et al., 1994) in a paper where they explain how to design an apparatus for doing compression tests. Tissue-culture medium is used in the experiment, is compressed at a given force, and is finally allowed to relax. During this process, the final relaxed force can be used for measuring the interfacial tension, thanks to video analysis of the shape of the compressed tissue. The values found, 8.3 and $4.3 \mathrm{dyn} / \mathrm{cm}$, respectively, from chick heart ventricle and liver verify $\gamma_{\text {heart }}>\gamma_{\text {liver. }}$. This explains previous results where embryonic heart tissue was engulfed by liver cells (Steinberg, 1993). Another example of the differential adhesion explanation is given 
(Beysens et al., 2000) using the same experiment. A better model for the force relaxation signal is found using a Kelvin model (Forgacs et al., 1998). Cells indeed relax like elastic materials in short times, but like liquids in longer times. The microscopic explanation of tissue compression is also outlined in their paper to explain how interfacial tension can rule important structural changes within the cellular structure. Rapid deformation of the cells (comparable to foams) first leads to affine elastic deformations, followed by reorganization of the cells, which relax in a liquid-like manner to their approximate initial shapes. Finally embryonic chick epithelial and neural cells are separated under reduced gravity, the same interfacial tension relationship being verified. This analysis of compression has recently been reconsidered (Palsson and Othmer, 2000) using a theoretical model, including both viscoelastic effects and signal transduction, adapted to the case of a dictyostelium discoideum population. This analysis explains how cells can reorganize themselves, and can also form star-shaped aggregates with no applied stress.

Tissue interfacial tensions thus arise from the interactions between the different cells and might also be important for explaining cell migration and sorting in phenomena like embryogenesis and angiogenesis. Their effect needs to be included to make tissue macroscopic models more accurate.

\section{Effective Medium Theory (EMT)}

The effective medium theory has been used mostly in the field of electrostatics (Choy, 1999) but is an interesting tool for accessing macroscopic laws once the proper assumptions are made at the microscopic level. Let us start with a continuum where inclusions (spherical first) are present. The basic idea is to say that the "field" felt by an inclusion is the macroscopic one, plus modifications, which arise from the rest of the inclusions, with the precise inclusion excluded. Consider, for example, the equation of motion of an isotropic elastic body, where $\boldsymbol{u}$ is the displacement field, which satisfies:

$$
\frac{\partial^{2} \mathbf{u}}{\partial t^{2}}=\mu \nabla^{2} \mathbf{u}+\left(K+\frac{\mu}{3}\right) \operatorname{grad}(\operatorname{div} \mathbf{u})
$$

where $K$ and $\mu$ are the compressibility and shear moduli, and these equations give rise to the usual hyperbolic wave equations with respective transverse and longitudinal velocities $\sqrt{\mu / \rho}$ and $\sqrt{(K+4 / 3 \mu) / \rho}$. Waves are solutions of the kind $\mathbf{u}=\mathbf{u}_{0} \exp i(\mathbf{k} \cdot \mathbf{r}-\omega t)$, and there is a dispersion relation between $\omega$ and $\mathbf{k}$. Two potentials, $\phi$ and $\psi$, related to $\mathbf{u}$, can be introduced which satisfy the Laplace equation $\nabla^{2} \phi=0, \nabla^{2} \psi=0$. Then we assume the long wave approximation, and follow calculus from Rayleigh, given scattering due to a sphere. This gives rise to asymptotic developments for the incident, scattered, and transmitted potentials. Following Yamakawa (1962), we find the unknown coefficients in front of the different potentials using the boundary conditions on the spherical inclusion. This leads to the following formula (called Maxwell-Garnett) for the moduli and density:

$$
\begin{aligned}
\frac{K_{2}-K}{3 K+4 \mu_{2}} & =c_{1} \frac{K_{2}-K_{1}}{3 K_{1}+4 \mu_{2}}, \\
\frac{\mu_{2}-\mu}{\mu+F_{2}} & =c_{1} \frac{\mu_{2}-\mu_{1}}{\mu_{1}+F_{2}}, \quad \rho_{2}-\rho_{1}=c_{1}\left(\rho_{2}-\rho_{1}\right)
\end{aligned}
$$

where $c_{1}$ is the volume fraction of the inclusions, and $K_{i}$, $\mu_{i}$ and $\rho_{i}$ are the moduli and densities in the $i$ th phase. The modulus $F_{i}$ is defined by:

$$
F_{i}=\frac{\mu_{i}}{6}\left[\frac{9 K_{i}+8 \mu_{i}}{K_{i}+2 \mu_{i}}\right] .
$$

Formula (31) is known as the Maxwell-Garnett formula but has a drawback. It does not predict a limiting regime, which is supposed to occur as concentration increases; this critical concentration is linked to the percolation threshold (Kirkpatrick, 1971). This threshold might be very important in particular when looking at a group of cells, which join each other and may be described as a percolating system. Note that Eq. (31) can be extended to the case where inclusions are not spheres, but ellipsoids. There is another formalism, know as the Bruggeman formalism, which is based on a slightly different approach, and this leads to the following results:

$$
\begin{aligned}
& \frac{1}{3 K+4 \mu}=\frac{c_{1}}{3 K_{1}+4 \mu}+\frac{1-c_{1}}{3 K_{2}+4 \mu}, \\
& \frac{1}{\mu+F}=\frac{c_{1}}{\mu_{1}+F}+\frac{1-c_{1}}{\mu_{2}+F} \\
& \rho=c_{1} \rho_{1}+\left(1-c_{1}\right) \rho_{2} .
\end{aligned}
$$

The advantage of this method is that it provides access to a critical volume fraction, which could correspond to a percolation threshold. Finally Eqs. (31-33) can be generalized, assuming the material is viscoelastic, the moduli being complex. This could be particularly interesting when looking at the dynamic response of viscoelastic tissues. No such analyses to model tissues have been found so far in the literature.

\section{Ensemble Averaging Methods}

The method of ensemble averaging has been introduced by Hashin (1964) for predicting the mechanical behavior of heterogeneous media but has led to interesting results in the field of suspension rheology, which is the example that we will analyze here. This has already been discussed briefly in the previous part on "suspensions". Consider a medium where particles 
(spherical for instance) are imbedded in a fluid matrix, as is usually the case for suspensions or emulsions. This is the problem studied by Batchelor (1970). The point is to determine the stress field in a system where particles are assumed to be force-free. The basic idea is to write the stress components as ensemble averages, where the average is taken over the ensemble of realizations for the suspension, i.e. the possible positions of particles. Other averages may be taken, which can enable less tedious calculations, but this method is usually the best for finding the bulk properties. Batchelor (1970) follows this argument, after writing the stress $\Sigma_{i j}$ as $\Sigma_{i j}=\overline{\sigma_{i j}}-\overline{\rho u_{i}^{\prime} u_{j}^{\prime}}$, thus taking into account the momentum flux across surfaces. Fluctuations correspond to the components of the velocity $\mathbf{u}^{\prime}$. Using the previous relation, one can average for the stress field $\Sigma_{i j}$ by integration over some well-chosen volume surrounding a sufficiently large number of particles. Then it is necessary to compute the flow field around the particle (either spherical or ellipsoidal) to put into the integral terms for stresses. Different solutions have been obtained for rigid particles or deformable particles (Taylor, 1932; 1934) so that the final averaged stress can be obtained and used for predicting different behaviors, like the viscosity of a suspension of force-free particles (Batchelor, 1970) or a suspension of spherical particles submitted to Brownian motion (Batchelor, 1977; see previous part on suspensions). Finally, the effect of Brownian motion on suspensions of non-spherical particles (Hinch and Leal, 1972) has also been determined similarly. The viscous stress was given in Eq. (23).

Applications to the rheology of blood are, therefore, possible. Deformable capsules in a Newtonian liquid have been investigated (Barthès-Biesel and Rallison, 1981) using two types of membranes (RBC or Mooney-Rivlin type of membrane). Finally, comparisons with experimental data (Drochon, 2003) have shown good agreement but the conclusions are that also using a membrane viscosity would improve the results.

Many applications of the method lie in the field of fluid mechanics, in particular the understanding of emulsions and suspensions.

\section{Discussion: Open Problems}

All the above theories are very interesting for modeling soft biological tissues, but they are still under way; in particular, people have tried to solve the easiest problems, although they are already quite challenging. From what has been said before, we may conclude this part by giving a list of problems, which may be of interest:

- attempt to find a macroscopic description of viscoelastic, viscoplastic systems;

- include the effect of interfacial tension to model cell interactions;

- consider the case of the intermediate concentration range (percolation);
- add a fluid or gel effect into a tensegrity or discrete homogenization method;

- include geometry, type, ECM, membrane, proteins characteristics, of the cell into the basic cell element for homogenization methods.

\section{MACROSCOPIC PROPERTIES: BIOLOGICAL MATERIALS}

\section{Tissue Rheological Properties}

Some of the most common biological tissues which have been characterized are bones, the heart muscle (cardiac muscle), and soft muscles, but discussions about other types of tissues are also found in the literature.

Bone can be considered to be an anisotropic composite material, made of collagen and hydroxyapatite. Its properties in the longitudinal direction are mainly elastic, and it can be said to be a common tissue. Its properties depend on the degree of humidity; it is found to be elastic when dry and exhibits a slightly plastic behavior when humid (Evans, 1969). A typical Young modulus, when subjected to uniaxial deformation, is around $18 \mathrm{GPa}$, and bone usually fails around $150 \mathrm{MPa}$. But in fact, its properties depend on the type (femur, tibia, humerus, radius, etc.). Bone is not a passive tissue, because it is constantly drained by blood, and cell remodeling is also to be accounted for. Its anisotropic properties have also been considered (see, for example, Lotz et al., 1991) and show two different privileged directions with different elastic moduli. Its viscoelastic nature has also been investigated, although it is not a major effect, but wet bone for instance may be considered as a viscoelastic material (Lakes et al., 1979; Lakes and Katz, 1979a,b). It has also been shown to exhibit a nonlinear regime before becoming an elastic material as in the case of demineralized bovine cortical bone (Bowman et al., 1996).

The heart muscle has also been investigated frequently in the literature. It is made of cardiac muscle fibers (actin and myosin filaments), organized in a branching interconnecting network, and contains capillaries and mitochondria, which produce oxygen and energy. Its viscoelastic properties have been analyzed in relaxation experiments (Pinto and Fung, 1973), where a normalized function can be introduced after a step stretch. The relaxation function is in fact a function of the stretch ratio, the $\mathrm{pH}$, the temperature and the chemical composition of the environment fluid. These parameters can indeed all have an effect on the reorganization of the actin and myosin filaments. When subject to uniaxial stretch, the force-extension curves exhibit the same behavior as that mentioned before in Eq. (29a) (collagen type), i.e. an exponential response (Fig. 10).

This equation is in fact typical of several tissues, but only when discussing 1D tests. The same authors also considered small-amplitude oscillatory experiments and found that the dynamic response increased very slowly as 


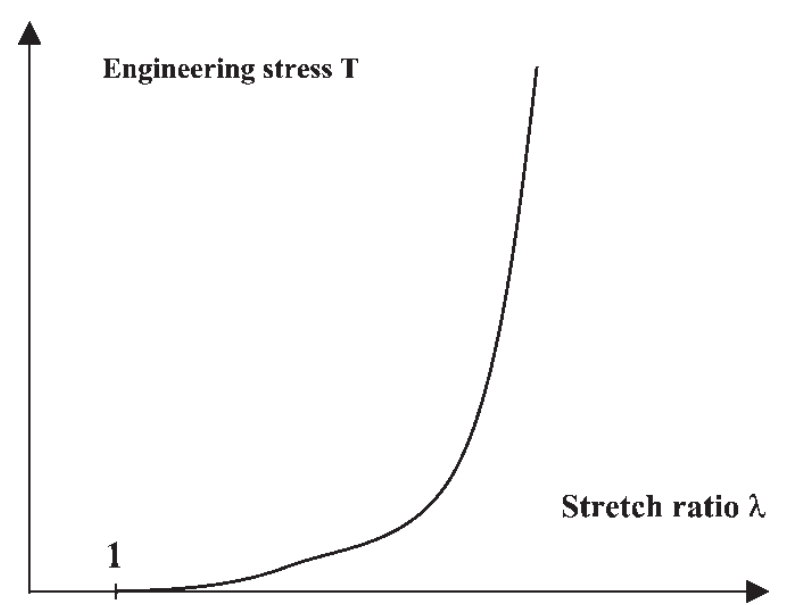

FIGURE 10 Typical engineering stress T vs. stretch ratio $\lambda$ of a soft tissue (muscle, vascular tissue, etc.) in a 1D test.

a function of frequency (over more than three decades). This may be considered to be similar to other viscoelastic solids such as rubbers, as discussed in the first part. Constitutive equations are then used, in particular the quasi-linear viscoelastic model (Fung, 1993a).

$$
T(t)=\int_{-\infty}^{t} G\left(t-t^{\prime}\right) \dot{T}^{e}\left(t^{\prime}\right) \mathrm{d} t^{\prime}
$$

where $T(t)$ is the engineering stress (referred to initial surface area) and $T^{e}\left(t^{\prime}\right)$ is called the elastic response corresponding to a step-stretch (see first part), and plays the role of the usual deformation, as in classical linear viscoelasticity. Although this formulation may be adapted to $3 \mathrm{D}$ cases, it is used more often with stretching or compressing 1D-assays. More work exists on the effect of $\mathrm{Ca}^{2+}$ on the capabilities of the heart muscle to retract; in particular contractile (or shortening) velocities have been measured relative to applied stress (Ross et al., 1966) and compared with Hill's theory (Hill, 1938). The latter theory predicts a law for the velocity of contraction as a decreasing function of the applied load in the muscle. Finally three-dimensional laws for the myocardium have been proposed, based on Eq. (13) and allowing for transverse anisotropy (Humphrey et al., 1990). They provide good agreement with the experiments.

Other types of soft muscles (intestine, vascular muscles, arteries, etc.) exhibit common features, and depend on the actin and myosin components, as well as ATP energy exchanges. Exponential laws like Eq. (29a) often approximate their stress-strain properties, although they might undergo irreversibility. Indeed, differences in loading and unloading regimes are observed in the case of ureteral tissues (Yin and Fung, 1971) or taenia coli muscles (Price et al., 1979). Similarly, Hill's equation (Hill, 1938) and relaxation data exhibit the same type of behavior, as compared to the myocardium.

To summarize, except for a few cases, all types of tissues are anisotropic and strain hardening Eq. (29a), exhibit a hysteresis in stress-strain curves, and relax to a non-zero equilibrium. Their microstructures are quite important and are usually chemically dependent on salts or ions $\left(\mathrm{Ca}^{2+}, \mathrm{K}^{+}, \mathrm{Na}^{+}\right)$and energy consumption is important for sustaining large stresses. Their highly resistant properties are due to the reinforcement by fibers (actin, myosin and collagen) which are elastic or nonlinear elastic components. Quasilinear theories like Eq. (34) are usually appropriate for predicting most behaviors, and the exponential dependence Eq. (29a) or the Mooney-Rivlin formulation Eq. (12) seems to work well. Finally, this concept is rather simple, but subtle differences may appear due to a velocity-dependent moduli. In general, in common oscillatory experiments, it is possible to check whether velocity is important or not. If not, one can use more general relations, such as the K-BKZ equation Eq. (20) or the generalized integral model Eq. (21). Finally, pseudostrain-energy functions are also a good means to model tissues, as shown in the case of skin (Tong and Fung, 1976). They indeed use a two-dimensional model adapted to the case of a membrane-like material, submitted to planar stresses. The model is in good agreement with the biaxial experiments (Lanir and Fung, 1974).

Dynamic tissue testing (see previous comment) has been carried out on a few occasions with special devices. In the first case, a shear oscillating device has been built (Arbogast et al., 1997) whereas conventional techniques are used in the second case (Chan, 2001). The latter example reveals that it might be possible to adapt the wellknown time-temperature superposition principle to some soft biological tissues, to access a larger range of frequencies. Therefore, one may be able to determine $G(t)$ from Eq. (34).

With regard to tissue growth, there are a number of important studies in the literature. Recently, the concept of growth has been analyzed rigorously in the framework on continuum theories (Humphrey and Rajagopal, 2002; Humphrey, 2003) by decomposition of the motion. This idea may be interesting for including a "live" contribution of the tissue (due to mechanotransduction, for example), as compared to inert models. Other research papers have investigated tumor growth, when the supply comes from nutrients (Byrne and Chaplain, 1997), assuming the tumor is a multicellular spheroid and using a continuum mechanics approach. Another approach by the same authors considers the influence of cell-cell interactions to improve the concept of tumor growth (Byrne and Chaplain, 1996). This idea is quite relevant to what was said in the part on "mathematical methods", because it takes into account the influence of membranes and interactions and may be a good way to model real tissues.

\section{Rheology of Biological Fluids}

\section{Blood Rheology}

Since the subject of blood rheology is wide, we will present here its main properties and try to correlate them with the models detailed in the first part. Blood is 
a suspension of cells inside a Newtonian fluid called plasma. Plasma is close to water with a viscosity slightly higher (of the other of $1 \mathrm{cP}$ ). The cells present inside the plasma are mainly erythrocytes (RBC), which have a biconcave shape (between 8 and $10 \mu \mathrm{m}$ in diameter), but there are also white cells (different types) and platelets. Hematocrit is the volume concentration of cells and is a very important quantity. Indeed, as the hematocrit concentration varies from 0 to say $90 \%$ (which is possible since cells are deformable), the properties of blood become non-Newtonian. Blood typically is a shear thinning fluid (Chien et al., 1966) but may be shown to exhibit a yield stress at very small rates, which is an increasing function of the hematocrit (Cokelet et al., 1963). This yield stress is attributed to interactions between the cells, which require strong forces necessary to create a flow field. The data of Cokelet et al. (1963) have been fitted successfully using a generalized viscous fluid law Eq. (15) and work remarkably well. The equation used for the 1D-shear stress is as follows:

$$
\sqrt{\sigma_{12}}=\sqrt{\sigma_{y}}+\sqrt{\eta \dot{\gamma}}
$$

This formula is called Casson's law (Casson, 1959) and was in fact derived for other types of suspensions. This equation may be considered in the flow of blood through a tube (representing a capillary). The exact solution reveals that the usual Poiseuille parabola profile is changed, in particular when the yield stress is increased. The flow exhibits a higher shear rate near the wall, whereas the central region shows an almost flat profile, in other words, it is close to plug flow. This is of course just a theoretical profile. In practice, due to the complex structure of blood, particular microscopic phenomena need to be considered, as that is the purpose of this article. When particles do not form aggregates, one can describe the suspension rheology (see parts on "suspensions" or "ensemble averaging") using models investigated by Batchelor (1970), Hinch and Leal (1972), Barthès-Biesel and Rallison (1981) and Drochon (2003). The formation of rouleaux (Goldsmith, 1972) is a well-known phenomenon which has been shown to affect the viscosity. Indeed, common relations for describing the viscosity of suspensions depend on the shape and concentration of particles. When particles form certain structures (rouleaux, for example), they may be responsible for increases in viscosity. The rouleaux behave like rod-like particles, whose motion has been shown to be described by Eq. (22), and can lead to tumbling motions, increased dissipations, and therefore higher viscosities.

Another important effect is the vessel dimension. Blood travels through various vessels going from the arteries (large diameter, order of biconcave) to microcapillaries where the diameter is of the order of a few microns. As the capillary size decreases, the apparent viscosity is shown to decrease. This is known as the Fahraeus-Linqvist effect (Fahraeus and Linqvist, 1931). Since we are not in the continuum model approximation (Batchelor, 1967), the argument of the increasing apparent shear rate (since the tube dimension is small) leading to decreasing viscosity of blood cannot be used. The explanation was provided by Barbee and Cokelet (1971) after observing that, when blood is made to flow through a narrow tube, it changes its microscopic properties by reducing the hematocrit. This means that fewer cells will travel through the tube or vessel, thus creating a decrease in viscosity.

Finally, the understanding of blood rheology is also affected by interactions between the different particles (or cells) which have been shown to form rouleaux, but interactions are also present at the wall with the endothelium lining and may affect blood flow through capillaries. These effects are also influenced by the high deformability of RBCs, which can actually go through 5- $\mu \mathrm{m}$-diameter capillaries. Similarly, white cells or cancer cells can be highly deformed and make their way through inter-endothelial junctions of about the same size. Such situations can only be analyzed in the context of microscopic rheology, and the presence of various proteins has a major influence on such interactions. Note that cellcell interactions can also lead to various pathological situations due to the formation of cell aggregates (blood clots, embolism).

\section{Other Types of Biological Fluids}

There are other types of fluids commonly encountered in the human body. Let us analyze a few of them. Synovial fluid, which appears in knee joints, has been analyzed using common rheometrical experiments (shearing tests mostly). The behavior is that of a non-Newtonian fluid, and can described by power-law models (as with polymers). The deviation from Newtonian fluids appears at shear rates of the order of $1 \mathrm{~Hz}$ as measured in oscillatory experiments (Balazs and Gibbs, 1970). Thus synovial fluids, like hyaluronic acids, can become less viscous at higher rates, allowing lubrication of knee joints.

Other types of fluids have also been characterized using oscillatory shearing tests, such as saliva and mucus. In these two cases, the measurements of the dynamic shear moduli $\left(G^{\prime}\right.$ and $\left.G^{\prime \prime}\right)$ reveal that these two fluids are highly viscoelastic, due to the presence of long polymer chains (DNA in the case of mucus), as was demonstrated in the first part. Considering rapid solicitations, the polymer chains cannot be uncoiled. However, for slow application of forces, the chains slide along each other, and the fluid exhibits viscous effects. At room temperature, these fluids show an almost constant elastic modulus $\left(G^{\prime}\right)$ and a slowly decreasing loss modulus $\left(G^{\prime \prime}\right)$ of the order of a few Pascals for dog mucus (Lutz et al., 1973) or a few tenths of a Pascal for saliva (Davis, 1973). This is typical of such viscoplastic fluids. Probably it is difficult to measure yield stresses for such fluids, but there is a tendency to believe in this property here, due to the presence of weak microscopic interactions. 


\section{CONCLUSIONS}

Modeling the rheological properties of biological tissues is a very difficult subject and is strongly dependent on the microscopic properties of individual cells. Without a complete description of the microstructure, it is impossible to build a constitutive equation. At present, there is a strong background in the modeling of viscoelastic and viscoplastic properties of polymers, suspensions and gels, which might be very useful for studying biological materials. In addition, the determination of local microrheological properties and cell interactions is now possible and should help produce a more detailed picture at the cellular level, in terms of its constituents.

We have shown that there are a variety of techniques to produce constitutive equations, when the cell properties are known. These techniques are not yet able to account for specific interactions between cells, individual behaviors, or special local properties, or to model the active response of a cell. This is a real challenge, which needs to be investigated further, for it would help to model the macroscopic behavior, i.e. the viscoelastic (or viscoplastic) nature of such biological materials.

Finally, the determination of macroscopic properties remains a challenge, due to the lack of data. Indeed such biological systems are hard to prepare and require the use of techniques that are not so well adapted. It is necessary to characterize them by combining different experiments under different conditions, so that every parameter from a given model can be obtained.

\section{References}

A-Hassan, E., Heinz, W.F., Antonik, M.D., D'Costa, N.P., Nageswaran, S., Schoenenberger, C.-A. and Hoh, J.H. (1998) "Relative microelastic mapping of living cells by atomic force microscopy", Biophys. J. 74, 1564-1578.

Alberts, B., Bray, D., Lewis, J., Raff, M., Roberts, K. and Watson, J.D. (1994) Molecular Biology of the Cell, 3rd Ed. (Garland Publishing, New York).

Arbogast, K.B., Thibault, K.L., Pinheiro, B.S., Winey, K.I. and Margulies, S.S. (1997) "A high-frequency shear device for testing soft biological tissues", J. Biomech. 30(7), 757-759.

Ashkin, A. and Dziedzic, J.M. (1989) "Internal cell manipulation using infrared laser traps", Proc. Natl Acad. Sci. USA 86, 7914-7918.

Balazs, E.A. and Gibbs, D.A. (1970) "The rheological properties and biological function of hyaluronic acid", In: Balazs, E.A., ed., Chemistry and Molecular Biology of the Intercellular Matrix (Academic Press, New York), Vol. 3, pp 1241-1253.

Barbee, J.H. and Cokelet, G.R. (1971) "The Fahraeus effect", Microvasc. Res. 34, 6-21.

Barthès-Biesel, D. and Rallison, J.M. (1981) "The time independent deformation of a capsule freely suspended in a linear flow", J. Fluid Mech. 113, 251.

Batchelor, G.K. (1967) An Introduction to Fluid Dynamics (Cambridge University Press, Cambridge).

Batchelor, G.K. (1970) "The stress system in a suspension of force-free particles", J. Fluid Mech. 41, 545-570.

Batchelor, G.K. (1977) "The effect of Brownian motion on the bulk stress in a suspension of spherical particles", J. Fluid Mech. 83, $97-117$.

Baumgaertel, M. and Winter, H.H. (1989) "Determination of discrete relaxation and retardation time spectra from dynamic mechanical data", Rheol. Acta 28, 511-519.
Bausch, A.R., Moëller, W. and Sackmann, E. (1999) "Measurement of local viscoelasticity and forces in living cells by magnetic tweezers", Biophys. J. 76, 573-579.

Bensoussan, A., Lions, J.L. and Papanicolau, G. (1978) Asymptotic Analysis for Periodic Structures (North-Holland, New York).

Berne, B.J. and Pecora, R. (1976) Dynamic Light Scattering: With Applications to Chemistry, Biology and Physics (Wiley, New York).

Beysens, D.A., Forgacs, G. and Glazier, J.A. (2000) "Cell sorting is analogous to phase ordering in fluids", Proc. Natl Acad. Sci. USA 97(17), 9467-9471.

Binnig, G., Quate, C.F. and Gerber, C. (1986) "Atomic force microscope", Phys. Rev. Lett. 56, 930-933.

Bird, R.B., Armstrong, R.C. and Hassager, O. (1987) Dynamics of Polymeric Liquids Fluid Mechanics, 2nd Ed. (Wiley Interscience, New York), Vol. 1.

Boehler, J.P. (1983) Mechanical Behaviour of Anisotropic Solids (Kluwer Academic Publishers, Dordrecht).

Bowman, S.M., Zeind, J., Gibson, L.J., Hayes, W.C. and McMahon, T.A. (1996) "The tensile behavior of demineralized bovine cortical bone", J. Biomech. 29(11), 1497-1501.

Byrne, H.M. and Chaplain, M.A.J. (1996) "Modelling the role of cellcell adhesion in the growth and development of carcinomas", Math. Comp. Modelling 24(12), 1-17.

Byrne, H.M. and Chaplain, M.A.J. (1997) "Free boundary value problems associated with the growth and development of multicellular spheroids", Eur. J. Appl. Math. 8(6), 639-658.

Caille, N., Thoumine, O., Tardy, Y. and Meister, J.-J. (2002) "Contribution of the nucleus to the mechanical properties of endothelial cells", J. Biomech. 35, 177-187.

Caillerie, D., Mourad, A. and Raoult, A. (2003) "Cell-to-muscle homogenization. Application to a constitutive law for the myocardium", Math. Model. Numer. Anal. 37(4), 681-698.

Canadas, P., Laurent, V.M., Oddou, C., Isabey, D. and Wendling, S. (2002) "A cellular tensegrity model to analyse the structural viscoelasticity of the cytoskeleton", J. Theor. Biol. 218, 155-173.

Canetta, E., Leyrat, A. and Verdier, C. (2003) "A physical model for predicting the adhesion between a functionalised microsphere and a living cell", Math. Comp. Modelling 37, 1121-1129.

Canetta, E. (2004) "Micromanipulation of living cells using an AFM spectrometer. Application to cancer", Ph.D. thesis (University of Grenoble I).

Casson, M. (1959) "A flow equation for pigment-oil suspensions of the printing ink type", In: Mills, C.C., ed., Rheology of Disperse Systems (Pergamon, Oxford), pp 84-104.

Chan, R.W. (2001) "Estimation of viscoelastic shear properties of vocalfold tissues based on time-temperature superposition", J. Acoust. Soc. Am. 110(3), 1548-1561.

Chien, S., Usami, S., Taylor, M., Lundberg, J.L. and Gregersen, M.I. (1966) "Effects of hematocrit and plasma proteins of human blood rheology at low shear rates", J. Appl. Physiol. 21, 81-87.

Chotard-Ghodsnia, R., Drochon, A., Duperray, A., Leyrat, A. and Verdier, C. (2003) "Static and dynamic interactions between circulating cells and the endothelium in cancer", In: Preziosi, L., ed., Cancer Modelling and Simulation, Chap. 9 (CRC Press, Boca Raton, FL), pp 243-267.

Choy, T.C. (1999) Effective Medium Theory. Principle and Applications (Oxford University Press, Oxford).

Clerc, L. (1976) "Directional differences of impulse spread in trabecular muscle from mammalian heart", J. Physiol. 255, 335-346.

Cokelet, G.R., Merrill, E.W., Gilliland, E.R., Shin, H., Britten, A. and Wells, R.E. (1963) "The rheology of human blood measurement near and at zero shear rate", Trans. Soc. Rheol. 7, 303-317.

Condeelis, J. (1993) "Life at the leading edge: the formation of cell protusions", Anпи. Rev. Cell Biol. 9, 411-444.

Crocker, J.C., Valentine, M.T., Weeks, E.R., Gisler, T., Kaplan, P.D., Yodh, A.G. and Weitz, D.A. (2000) "Two-point microrheology of inhomogeneous soft materials", Phys. Rev. Lett. 85, 888-891.

Dasgupta, B.R., Tee, S.-Y., Crocker, J.C., Frisken, B.J. and Weitz, D.A. (2002) "Microrheology of poly-ethylene oxide using diffusing wave spectroscopy and single scattering", Phys. Rev. E 65, 051505.

Davis, S. (1973) In: Gabelnick, H.L. and Litt, M., eds, Rheology of Biological Systems (Thomas, C.C., Springfield, IL), pp 158-194.

Divet, F., Biben, T., Cantat, I., Stephanou, A., Fourcade, B. and Misbah, C. (2002) "Fluctuations of a membrane interacting with a diffusion field", Europhys. Lett. 60(5), 795-801.

Doi, M. and Edwards, S. (1986) The Theory of Polymer Dynamics (Oxford University Press, New York). 
Doi, M. and Ohta, T. (1991) "Dynamics and rheology of complex interfaces. I", J. Chem. Phys. 95(2), 1242-1248.

Drochon, A. (2003) "Rheology of dilute suspensions of red blood cells: experimental and theoretical approaches", Eur. Phys. J. AP 22, $155-162$.

Einstein, A. (1906) "Eine neue Bestimmung der Moleküldimension", Ann. Phys. 19, 289-306.

Einstein, A. (1911) "Berichtigung zu meiner Arbeit: Eine neue Bestimmung der Moleküldimensionen", Ann. Phys. 34, 591-592.

Evans, F.G. (1969) "The mechanical properties of bone", Artificial Limbs 13, 37-48.

Evans, E. (1973) "New membrane concept applied to the analysis of fluid shear- and micropipette-deformed red blood cells", Biophys. J. 13, 941-954.

Evans, E. and Skalak, R. (1980) Mechanics and Thermodynamics of Biomembranes (CRC Press, Boca Raton, FL).

Evans, E. and Yeung, A. (1989) "Apparent viscosity and cortical tension of blood granulocytes determined by micropipette aspiration", Biophys. J. 56, 151-160.

Fabry, B., Maksym, G.N., Butler, J.P., Glogauer, M., Navajas, D. and Fredberg, J.J. (2001) "Scaling the microrheology of living cells", Phys. Rev. Lett. 87(14), 148102.

Fahraeus, R. and Linqvist, T. (1931) "Viscosity of blood in narrow capillary tubes", Am. J. Physiol. 96, 562-568.

Flory, P.J. (1953) Principles of Polymer Chemistry (Cornell University Press, Cornell).

Forgacs, G., Foty, R.A. and Steinberg, M.S. (1998) "Viscoelastic properties of living embryonic tissues: a quantitative study", Biophys. J. 74, 2227-2234.

Foty, R.A., Forgacs, G., Pfleger, C.M. and Steinberg, M.S. (1994) "Liquid properties of embryonic tissues: measurement of interfacial tensions", Phys. Rev. Lett. 72(14), 2298-2301.

Fuller, R.B. (1961) “Tensegrity”, Portfolio Artnews Annual 4, 112-127.

Fung, Y.C. (1988) "Microrheology and constitutive equation of soft tissue", Biorheology 25(1-2), 261-270.

Fung, Y.C. (1993a) Biomechanics. Mechanical Properties of Living Tissues, 2nd Ed. (Springer-Verlag, New York).

Fung, Y.C. (1993b) First Course in Continuum Mechanics, 3rd Ed. (Prentice Hall)

de Gennes, P.G. (1979) Scaling Concepts in Polymer Physics (Cornell University Press, Ithaca, NY).

Gibson, L.J. and Ashby, M. (1988) Mechanics of Cellular Solids (Pergamon Press, Oxford).

Giesekus, H. (1966) "Die Elastizität von Flüssigkeiten", Rheol. Acta 5, $29-35$.

Gittes, F., Schnurr, B., Olmsted, P.D., MacKintosh, F.C. and Schmidt, C.F. (1997) "Microscopic viscoelasticity: shear moduli of soft materials measured by thermal fluctuations", Phys. Rev. Lett. 79(17), $3286-3289$.

Goldsmith, H.L. (1972) "The microrheology of human erythrocyte suspensions", In: Becker, E. and Mikhailov, G.K., eds, Theoretical and Applied Mechanics Proc. 13th IUTAM Congress (Springer, New York).

Grace, H.P. (1982) "Dispersion phenomena in high viscosity immiscible fluid systems and application of static mixers as dispersion devices in such systems", Chem. Eng. Commun. 14, 225-277.

Guenet, J.M. (1992) Thermoreversible Gelation of Polymers and Biopolymers (Academic Press, London).

Hashin, Z. (1964) "Theory of mechanical behaviour of heterogeneous media", Appl. Mech. Rev. 17, 1-9.

He, X. and Dembo, M. (1997) "A dynamic model of cell division", In: Alt, W., Deutsch, A. and Dunn, G., eds, Mechanisms of Cell and Tissue Motion (Birkhaüser Verlag, Basel), pp 55-66.

Helfer, E., Harlepp, S., Bourdieu, L., Robert, J., MacKintosh, F.C. and Chatenay, D. (2001) "Viscoelastic properties of actin-coated membranes", Phys. Rev. E 63, 021904.

Helfrich, W. (1973) "Elastic properties of lipid bilayers: theory and possible experiments", Z. Naturforsch. C28, 693-703.

Hénon, S., Lenormand, G., Richter, A. and Gallet, F. (1999) "A new determination of the shear modulus of the human erythrocyte membrane using optical tweezers", Biophys. J. 76, $1145-1151$.

Hertz, H. (1896) Miscellaneous Papers (MacMillan and Co., London), pp $146-183$.

Hill, A.V. (1938) "The abrupt transition from rest to activity in muscle", Proc. R. Soc. Lond. B 126, 136-195.
Hinch, E.J. and Leal, L.G. (1972) "The effect of Brownian motion on the rheological properties of a suspension of non-spherical particles", J. Fluid Mech. 52, 683-712

Hochmuth, R.M. (2000) "Review. Micropipette aspiration of living cells", J. Biomech. 33, 15-22.

Hochmuth, R.M. and Marcus, W.D. (2002) "Membrane tethers formed from blood cells with available area and determination of their adhesion energy", Biophys. J. 82, 2964-2969.

Humphrey, J.D. (2003) "Continuum biomechanics of soft biological tissues", Proc. R. Soc. Lond. A 459, 3-46.

Humphrey, J.D. and Rajagopal, K.R. (2002) "A constrained mixture model for growth and remodeling of soft tissues", Math. Models Meth. Appl. Sci. 12, 407-430.

Humphrey, J.D., Strumpf, R.H. and Yin, F.C.P. (1990) "Determination of a constitutive relation for passive myorcardium, I. A nero-functional form, II. Parameter identification", J. Biomech. Eng. 112, 333-339, See also $340-346$.

Ingber, D.E. (1993) "Cellular tensigrity: defining new rules of biological design that govern the cytoskeleton", J. Cell Sci. 104, 613-627.

Ingber, D.E. and Jamieson, J.D. (1982) "Tumor formation and malignant invasion: role of basal lamina", In: Liotta, L.A. and Hart, I.R., eds, Tumor Invasion and Metastasis (Martinus Nijhoff, The Hague, Netherlands), pp 335-357.

Ingber, D.E., Madri, J.A. and Jamieson, J.D. (1981) "Role of basal lamina in the neoplastic disorganization of tissue architecture", Proc. Natl Acad. Sci. USA 78, 3901-3905.

Ingber, D.E., Heidemann, S.R., Lamoureux, P. and Buxbaum, R.E. (2000) "Opposing views on tensegrity as a structural framework for understanding cell mechanics", J. Appl. Physiol. 89, 1663-1678.

Israelachvili, J. (1992) Intermolecular and Surface Forces, 2nd Ed. (Academic Press, London).

Jackson, J.K. and Winter, H.H. (1996) "The relaxation of linear flexible polymers which are slightly poly-disperse", Rheol. Acta 35, $645-655$.

Jeffery, G.B. (1922) "The motion of ellipsoidal particles immersed in a viscous fluid", Proc. R. Soc. Lond. A 102, 161-179.

Johnson, M.W. and Segalman, D. (1977) "A model for viscoelastic fluid behavior which allows non-affine deformation", J. Non-Newtonian Fluid Mech. 2(3), 225-270.

Johnson, K.L., Kendall, K. and Roberts, A.D. (1971) "Surface energy and the contact of elastic solids", Proc. R. Soc. Lond. A 324, 301-313.

Kirkpatrick, S. (1971) "Classical transport in disordered media: scaling and effective medium theories", Phys. Rev. Lett. 27(25), 1722-1725.

Lakes, R.S. and Katz, J.L. (1979a) "Viscoelastic properties of wet cortical bone. II. Relaxation mechanisms", J. Biomech. 12, 679-687.

Lakes, R.S. and Katz, J.L. (1979b) "Viscoelastic properties of wet cortical bone. III. A nonlinear constitutive equation", J. Biomech. 12, 689-698.

Lakes, R.S., Katz, J.L. and Sternstein, S. (1979) "Viscoelastic properties of wet cortical bone. I. Torsional and biaxial studies", J. Biomech. 12, $657-678$

Landau, L.D. and Lifshitz, E.M. (1959) Theory of Elasticity (Mir Editions, Moscow).

Lanir, Y. and Fung, Y.C. (1974) "Two-dimensional mechanical properties of rabbit skin. I. Experimental system, II. Experimental results", J. Biomech. 7, 29-34, See also 171-182.

Larson, R.G. (1988) Constitutive Equations for Polymer Melts and Solutions (Butterworths, Boston).

Larson, R.G. (1999) The Structure and Rheology of Complex Fluids (Oxford University Press, New York).

Laun, H.M. (1984) "Rheological properties of aqueous polymer dispersions", Angew. Makromol. Chem. 124/125, 335-359.

Lee, J., Choi, J.B. and Choi, K. (1996) "Application of homogenization FEM analysis to regular and re-entrant honeycomb structures", J. Mater. Sci. 31, 4105-4110.

Leonov, A.I. (1976) "Nonequilibrium thermodynamics and rheology of viscoelastic polymer media", Rheol. Acta 15(2), 85-98

Leyrat, A., Duperray, A. and Verdier, C. (2003) "Adhesion mechanisms in cancer metastasis", In: Preziosi, L., ed., Cancer Modelling and Simulation, Chap. 8 (CRC Press), pp 221-242.

Lipowsky, R. and Sackmann, E. (1995) Structure and Dynamics of Membranes Handbook of Biological Physics (Elsevier Science, Amsterdam), Vol. 1A-1B.

Lotz, J.C., Gerhart, T.N. and Hayes, W.C. (1991) "Mechanical properties of metaphysical bone in the proximal femur", J. Biomech. 24 , $317-329$. 
Lutz, R.J., Litt, M. and Chakrin, L.W. (1973) "Physical-chemical factors in mucus rheology", In: Gabelnick, H.L. and Litt, M., eds, Rheology of Biological Systems (Thomas, C.C., Springfield, IL), pp 119-157.

Macosko, C.W. (1994) Rheology, Principles, Measurements and Applications (Wiley-VCH, New York).

Mahaffy, R.E., Shih, C.K., MacKintosh, F.C. and Käs, J. (2000) "Scanning probe-based frequency dependent microrheology of polymer gels and biological cells", Phys. Rev. Lett. 85(4), 880-883.

Mason, T.G. and Weitz, D.A. (1995) "Optical measurements of the frequency-dependent linear viscoelastic moduli of complex fluids", Phys. Rev. Lett. 74, 1250-1253.

Mason, T.G., Ganesan, K., van Zanten, J.H., Wirtz, D. and Kuo, S.C. (1997) "Particle tracking microrheology of complex fluids", Phys. Rev. Lett. 79, 3282-3285.

McGrath, J.L., Hartwig, J.H. and Kuo, S.C. (2000) "The mechanics of F-actin microenvironments depend on the chemistry of probing surfaces", Biophys. J. 79, 3258-3266.

Neu, J.C. and Krassowska, W. (1993) "Homogenization of syncytial tissues", Crit. Rev. Biomed. Eng. 21(2), 137-199.

Neves, M.M., Rodrigues, H. and Guedes, J.M. (2000) "Optimal design of periodic linear elastic microstructures", Comput. Struct. 76, $421-429$.

Oldroyd, J.G. (1953) "The elastic and viscous properties of emulsions and suspensions", Proc. R. Soc. Lond. A 218, 122-132.

Oldroyd, J.G. (1955) "The effect of interfacial stabilizing films on the elastic and viscous properties of emulsions", Proc. R. Soc. Lond. A 232, 567-577.

Palecek, S.P., Loftus, J.C., Ginsberg, M.H., Laufenberger, D.A. and Horwitz, A.F. (1997) "Integrin-ligand binding properties govern cellmigration speed through cell-substratum adhesiveness", Nature 385, $537-540$.

Palierne, J.-F. (1990) "Linear Rheology of viscoelastic emulsions with interfacial tension", Rheol. Acta 29, 204-214.

Palsson, E. and Othmer, H.G. (2000) "A model for individual and collective cell movement in dictyostelium discoideum", Proc. Natl Acad. Sci. USA 97(19), 10448-10453.

Perkins, T.T., Smith, D.E., Larson, R.G. and Chu, S. (1995) "Stretching of a single tethered polymer in a uniform flow", Science 268, 83-87.

Phan-Thien, N. and Tanner, R.I. (1977) "A new constitutive equation derived from network theory", J. Non-Newtonian Fluid Mech. 2 , $353-365$.

Pignon, F., Magnin, A. and Piau, J.-M. (1997) "Butterfly light scattering pattern and rheology of a sheared thixotropic clay gel", Phys. Rev. Lett. 79(23), 4689-4692.

Pine, D.J., Weitz, D.A., Chaikin, P.M. and Herbolzheimer, E. (1988) "Diffusing wave spectroscopy", Phys. Rev. Lett. 60, 1134-1137.

Pinto, J.G. and Fung, Y.C. (1973) "Mechanical properties of stimulated papillary muscle in the passive state", J. Biomech. 6, $596-616$.

Price, J.M., Patitucci, P. and Fung, Y.C. (1979) "Mechanical properties of resting taenia coli smooth muscle", Am. J. Physiol. 236, 211-220.

Ross, Jr, J., Covell, J.W. and Factor, S.M. (1966) "Contractile state of the heart", Circulation Res. 18, 149-163.

Safran, S.A. (1994) Statistical Thermodynamics of Surfaces, Interfaces, and Membranes (Addison-Wesley, Reading, MA).

Sanchez-Palencia, E. (1980) Non Homogeneous Media and Vibration Theory (Springer-Verlag, Berlin), Vol. 127, Lectures Notes in Physics.

Schmidt, F.G., Ziemann, F. and Sackmann, E. (1996) "Shear field mapping in actin networks by using magnetic tweezers", Eur. Biophys. J. 24, 348-353.

Schmidt, F.G., Hinner, B. and Sackmann, E. (2000a) "Microrheometry underestimates the viscoelastic moduli in measurements on F-actin solutions compared to macrorheometry", Phys. Rev. E. 61(5), $5646-5653$.

Schmidt, F.G., Hinner, B., Sackmann, E. and Tang, J.X. (2000b) "Viscoelastic properties of semiflexible filamentous bacteriophage fd", Phys. Rev. E. 62(4), 5509-5517.

Schnurr, B., Gittes, F., MacKintosh, F.C. and Schmidt, C.F. (1997) "Determining microscopic viscoelasticity in flexible and semiflexible polymer networks from thermal fluctuations", Macromolecules 30, $7781-7792$

Sedov, L. (1975) Continuum Mechanics (Mir editions, Moscow) Vols. 1 and 2.

Sheetz, M. (1998) Laser Tweezers in Cell Biology (Academic Press, San Diego).
Skalak, R. (1973) "Modelling the mechanical behavior of red blood cells", Biorheology 10, 229-238.

Skalak, R., Tozeren, A., Zarda, R.P. and Chien, S. (1973) "Strain energy functions of red blood cell membranes", Biophys. J. 13, 245-264.

Smith, G.F. (1994) Constitutive Equations for Anisotropic and Isotropic Materials (North-Holland, Amsterdam).

Sollich, P., Lequeux, F., Hébraud, P. and Cates, M.E. (1997) "Rheology of soft glassy materials", Phys. Rev. Lett. 78(10), 2020-2023.

Sollich, P. (1998) "Rheological constitutive equation for a model of soft glassy materials", Phys. Rev. E 58(1), 738-759.

Steinberg, M.S. (1993) In: Beysens, D., Bocara, N. and Forgacs, G., eds, Dynamical Phenomena at Interfaces, Surfaces and Membranes (Nova Science Publishers, Commack, NY).

Taylor, G.I. (1932) "The viscosity of a fluid containing small drops of another fluid", Proc. R. Soc. A 138, 41-48.

Taylor, G.I. (1934) "The formation of emulsions in definable fields of flow", Proc. R. Soc. A 146, 501-523.

Thoumine, O. and Ott, A. (1997) "Time scale dependent viscoelastic and contractile regimes in fibroplasts probed by microplate manipulation", J. Cell Sci. 110, 2109-2116.

Tollenaere, H. and Caillerie, D. (1998) "Continuous modeling of lattice structures by homogenization", Adv. Eng. Software 29(7-9), 699-705.

Tong, P. and Fung, Y.C. (1976) "The stress-strain relationship for the skin", J. Biomech. 9, 649-657.

Tran-Son-Tay, R., Kan, H.C., Udaykumar, H.S., Damay, E. and Shyy, W. (1998) "Rheological modelling of leukocytes", Med. Biol. Eng. Comput. 36, 246-250.

Tseng, Y. and Wirtz, D. (2001) "Mechanics and multiple particle tracking microheterogeneity of $\alpha$-actinin-crosslinked actin filament networks", Biophys. J. 81, 1643-1656.

Verdier, C. (2001) "The influence of the viscosity ratio on polymer droplet collision in quiescent blends", Polymer 42(16), 6999-7007.

Verdier, C. and Piau, J.-M. (2003) "The effect of the nonlinear viscoelastic properties on tack", J. Polym. Sci. Part B: Polym. Phys. 41(23), 3139-3149.

Verdier, C., Longin, P.-Y. and Piau, M. (1998) "Dynamic shear and compressional behavior of polydimethylsiloxanes: ultrasonic and low frequency characterization", Rheol. Acta 37(3), 234-244.

Verdier, C., Jin, Q., Singh, P., Leyrat, A., Chotard-Ghodsnia, R. and Duperray, A. (2003) "Modeling the rolling and deformation of a circulating cell adhering on an adhesive wall under flow", Arch. Physiol. Biochem. 111, 14

Wagner, M.H. (1990) "A constitutive analysis of extensional flows of polyisobutylene", J. Rheol. 34(6), 943-958.

Walters, K. (1975) Rheometry (Chapman \& Hall, London).

Wang, N., Butler, J.P. and Ingber, D.E. (1993) "Mechanotransduction across the cell surface and through the cytoskeleton", Science 260, $1124-1127$.

Wang, N., Naruse, K., Stamenovic, D., Fredberg, J.J., Mijailovich, S.M., Tolic-Norrelikke, I.M., Polte, T., Mannix, R. and Ingber, D.E. (2001) "Mechanical behavior of living cells consistent with the tensegrity model", Proc. Natl Acad. Sci. USA 98(14), 7765-7770.

Weaire, D. and Hutzler, S. (1999) The Physics of Foams, 2nd Ed. (Clarendon Press, New York).

Wendling, S., Oddou, C. and Isabey, D. (1999) "Stiffening response of a cellular tensegrity model", J. Theor. Biol. 196, 309-325.

Wendling, S., Oddou, C. and Isabey, D. (2000) "Approche structurale de la mécanique du cytosquelette. Solide alvéolaire vs. modèle de tenségrité", C. R. Acad. Sci. IIb. t328, 97-104.

White, J.L. and Metzner, A.B. (1963) "Development of constitutive equations for polymeric melts and solutions", J. Appl. Polym. Sci. 7, $1867-1889$.

Winter, H.H. and Chambon, F. (1986) "Analysis of linear viscoelasticity of a crosslinking polymer at the gel point", J. Rheol. 30(2), 367-382.

Wojcikiewicz, E.P., Zhang, X., Chen, A. and Moy, V.T. (2003) "Contributions of molecular binding events and cellular compliance to the modulation of leukocyte adhesion", J. Cell. Sci. 116(12), $2531-2539$.

Yamada, S., Wirtz, D. and Kuo, S.C. (2000) "Mechanics of living cells measured by laser tracking microrheology", Biophys. J. 78, 1736-1747.

Yamakawa, N. (1962) "Scattering and attenuation of elastic waves", Geophys. Mag. (Tokyo) 31, 63-103.

Yeung, A. and Evans, E. (1989) "Cortical shell-liquid core model for passive flow of liquid-like spherical cells into micropipets", Biophys. J. 56, 139-149.

Yin, F.C.P. and Fung, Y.C. (1971) "Mechanical properties of isolated mammalian ureteral segments", Am. J. Physiol. 221, 1484-1493. 


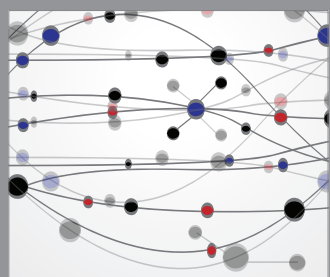

The Scientific World Journal
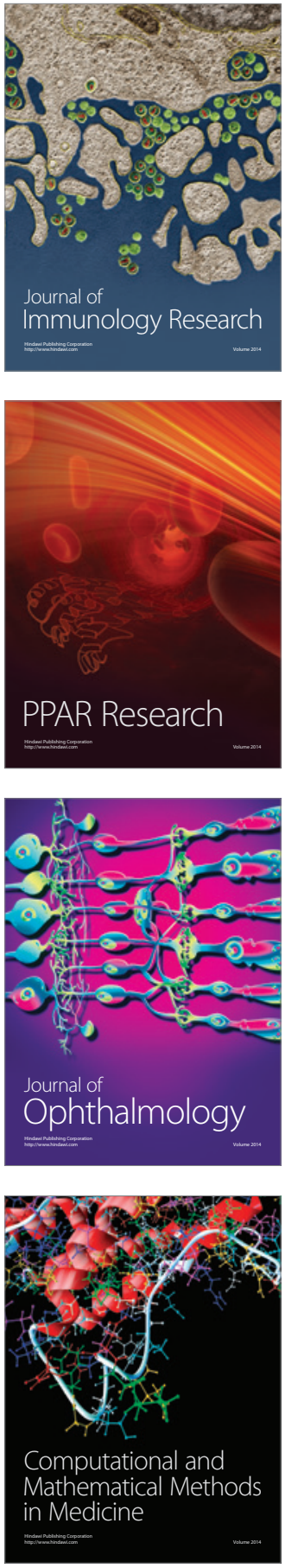

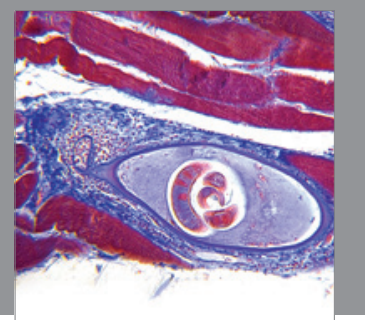

Gastroenterology

Research and Practice
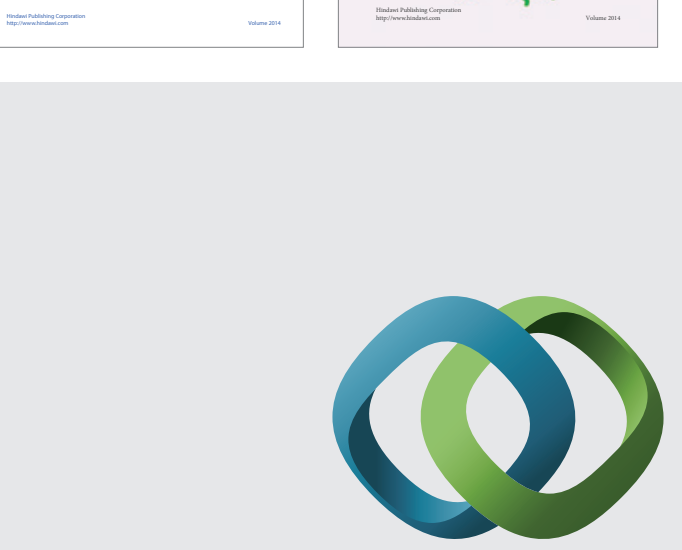

\section{Hindawi}

Submit your manuscripts at

http://www.hindawi.com
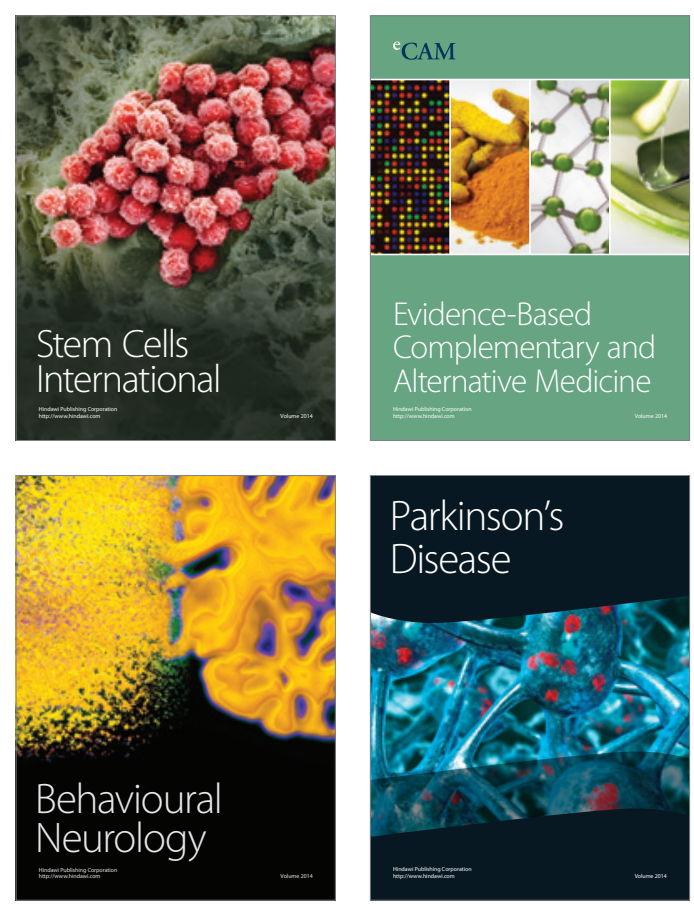

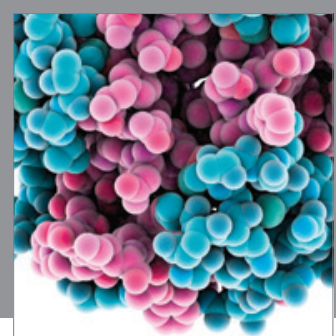

Journal of
Diabetes Research

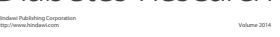

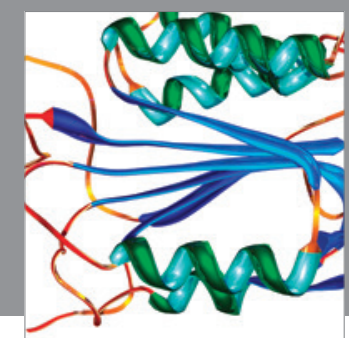

Disease Markers
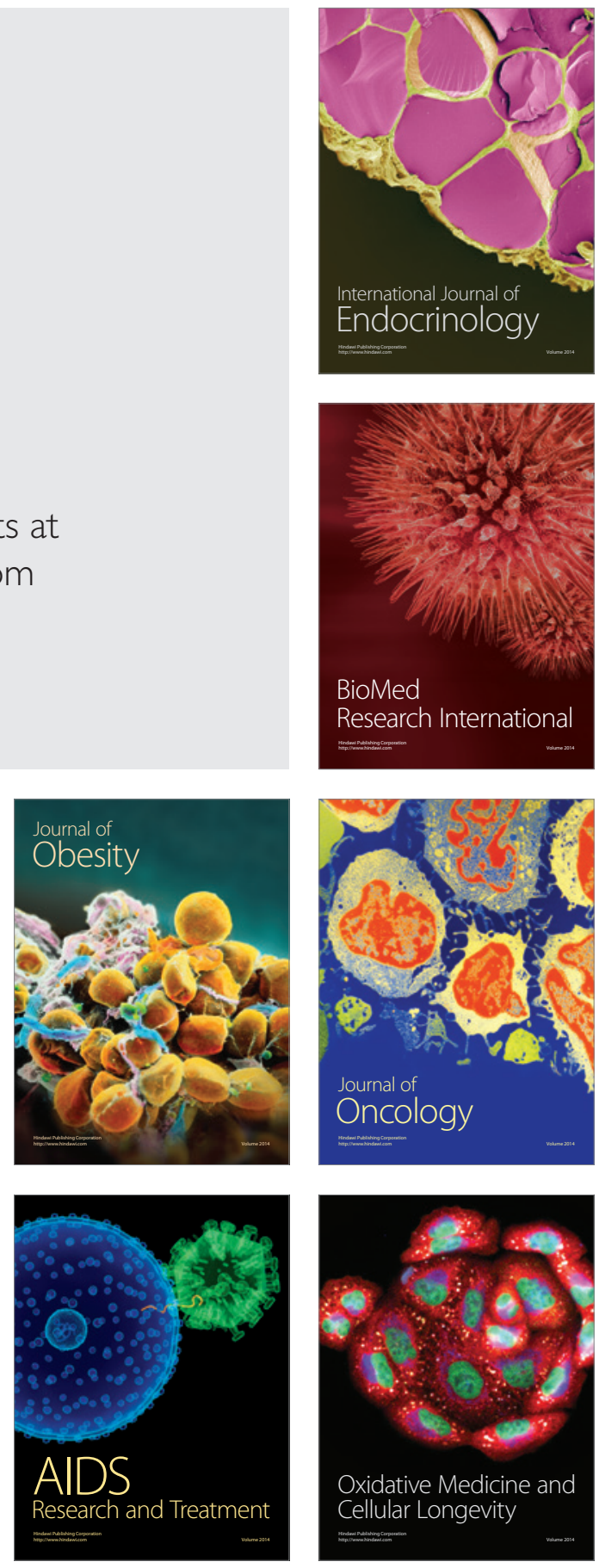